\title{
Positive Hankel operators, positive definite kernels and related topics
}

\section{Piotr Niemiec ${ }^{1}$}

Received: 18 January 2020 / Accepted: 9 March 2020/Published online: 25 March 2020

(C) The Author(s) 2020. This article is published with open access at Springerlink.com

\begin{abstract}
It is shown that a positive (bounded linear) operator on a Hilbert space with trivial kernel is unitarily equivalent to a Hankel operator that satisfies the double positivity condition if and only if it is non-invertible and has simple spectrum (that is, if this operator admits a cyclic vector). More generally, for an arbitrary positive (bounded linear) operator $A$ on a Hilbert space $H$ with trivial kernel the collection $\mathscr{V}(A)$ of all linear isometries $V: H \rightarrow H$ such that $A V$ is positive as well is investigated. In particular, operators $A$ such that $\mathscr{V}(A)$ contains a pure isometry with a given deficiency index are characterized. Some applications to unbounded positive selfadjoint operators as well as to positive definite kernels are presented. In particular, positive definite matrix-type square roots of such kernels are studied and kernels that have a unique such root are characterized. The class of all positive definite kernels that have at least one such a square root is also investigated.
\end{abstract}

Keywords Hankel operator · Double positivity condition · Positive operator $\cdot$ Positive definite kernel $\cdot$ Positive square root $\cdot$ Operator range

Mathematics Subject Classification 47B35 - 46E22 - 47B25

\section{Communicated by Martin Mathieu.}

Dedicated to Professor Franciszek Hugon Szafraniec - the main of my two scientific fathers, on the occasion of his 80th birthday.

Piotr Niemiec

piotr.niemiec@uj.edu.pl

1 Wydział Matematyki i Informatyki, Uniwersytet Jagielloński, ul. Łojasiewicza 6, 30-348 Kraków, Poland 


\section{Introduction}

In [15] the authors characterized (in the language of the multiplicity theory of separable Hilbert space self-adjoint operators) bounded self-adjoint operators that are unitarily equivalent to Hankel. This is a deep result whose proof is difficult and long. For a bounded positive operator $A$ on a separable Hilbert space the following two theorems immediately follow:

- if $A$ is unitarily equivalent to a Hankel operator, the essential supremum of the multiplicity function of $A$ does not exceed 2;

- if the essential supremum of the multiplicity function of $A$ does not exceed $1, A$ is unitarily equivalent to a Hankel operator.

Recall that the essential supremum of the multiplicity function of a bounded selfadjoint operator $A$ acting on a separable Hilbert space does not exceed $n \in$ $\{1,2, \ldots\}$ iff $A$ can be decomposed as the direct sum of at most $n$ self-adjoint operators each of which has a cyclic vector.

Hankel operators can be defined in a few equivalent ways. One of them, appropriate to our investigations, reads as follows: a bounded operator $A: \ell_{2} \rightarrow \ell_{2}$ is Hankel if $A S=S^{*} A$ where $S$ is the standard unilateral shift (that is, $S$ is a linear isometry satisfying $S e_{n}=e_{n+1}$ for any $n \geq 0$ where $e_{0}, e_{1}, \ldots$ is the canonical orthonormal basis of $\ell_{2}$ ). So, $A$ is both Hankel and self-adjoint iff both $A$ and $A S$ are self-adjoint. When in the last condition we replace the adjective self-adjoint by positive, we obtain the so-called double positivity condition [13]: $A$ is a Hankel operator satisfying the double positivity condition if both $A$ and $A S$ are positive. Having this notion in mind, a natural question (related to the main topic of the aforementioned paper) arises of when a (bounded) positive operator on a separable Hilbert space is unitarily equivalent to a Hankel operator satisfying the double positivity condition. In the present paper we answer this question under the additional assumption that the operator in question has trivial kernel. Our main result reads as follows (below $\mathcal{R}(T)$ denotes the range of an operator $T$ ).

Theorem 1 For a positive bounded operator $A: H \rightarrow H$ with trivial kernel and a cardinal $\mathrm{m}>0$ the following conditions are equivalent:

(i) there exists a pure isometry $V: H \rightarrow H$ such that $A V$ is positive and

$$
\operatorname{dim} \mathcal{R}(V)^{\perp}=\mathfrak{m} ;
$$

(ii) A is non-invertible, $\operatorname{dim}(H)=\max \left(\mathrm{m}, \aleph_{0}\right)$ and an appropriate condition of the following three is fulfilled:

( $\alpha) \quad m<\aleph_{0}$ and the essential supremum of the multiplicity function of A does not exceed $\mathrm{m}$; or

( $\beta) \quad \mathrm{m}=\aleph_{0} ;$ or

$(\gamma) \quad m>\aleph_{0}$ and there exists a closed linear subspace $Z$ of $H$ such that $Z \cap$ $\mathcal{R}(A)=\{0\}$ and $\operatorname{dim}(Z)=\mathrm{m}$. 
In particular, a bounded one-to-one linear operator is unitarily equivalent to a Hankel operator that satisfies the double positivity condition iff it is positive, noninvertible and has simple spectrum.

Theorem 1 shows (in particular) that for infinite $m$ the property (i) above depends only on the range of the operator $A$ (i.e., if two positive operators $A$ and $B$ have trivial kernels and their ranges coincide, then either both $A$ and $B$ satisfy (i) or none of them). In Theorem 5 below we gather conditions on a dense operator range $\mathcal{R}$ in a Hilbert space $H$ related to the foregoing statement $(\gamma)$-that is, conditions equivalent to the existence of a closed linear subspace $Z$ of $H$ such that $Z \cap \mathcal{R}=$ $\{0\}$ and $\operatorname{dim}(Z)=\operatorname{dim}(H)$.

Our proofs are independent of the results from [15] and are much simpler. We use basics of the operator theory and of the spectral theory of self-adjoint operators.

Another topic we deal with in this paper is related to Hilbert space reproducing (that is, positive definite) kernels. They are a useful tool in both Hilbert space theory and complex analysis (where they are known as Bergman kernels). Since the seminal paper of Aronszajn [2], positive definite kernels are a subject of an intrinsic theory. Although Bergman [3,4] is considered by a sizeable mathematical community as the father of that theory, it is Zaremba's work [25], published 15 years earlier than the first Bergman's on kernels, where the reproducing property (without any name) appeared for the first time-see, e.g., [2] or [23]. We wish to emphasize Zaremba's contribution to the theory by calling him its forefather.

As positive definite kernels naturally generalize positive matrices (for complex square matrices can be seen as kernels defined on finite sets), it is natural to investigate various properties of such matrices and recognize those of them that inhere in all such kernels. In the present paper (in Sect. 5) we characterize those kernels which have the so-called matrix-type square root. To be more precise, we introduce the following

Definition 1 Let $K: X \times X \rightarrow \mathbb{C}$ and $L: X \times X \rightarrow \mathbb{C}$ be two positive definite kernels. $K$ is said to be a positive definite matrix-type square root (for short: a pdms root) of $L$ if for all $x, z \in X$ :

$$
L(x, z)=\sum_{y \in X} K(x, y) K(y, z) .
$$

(More on the above notion can be found in Sect. 5.) A classical result from matrix theory (or, more generally, from bounded Hilbert space operator theory) says that any positive matrix has a unique positive square root. A natural question arises as to how far this result extends in the realm of reproducing kernels. Our main result in this direction reads as follows. (Below we write " $K \ll L$ " to express that $L-K$ is a positive definite kernel; and $\delta_{X}$ is a kernel on $X$ such that $\delta_{X}(x, y)=1$ if $y=x$ and $\delta_{X}(x, y)=0$ otherwise.)

Theorem 2 For a positive definite kernel $K: X \times X \rightarrow \mathbb{C}$ the following conditions are equivalent: 
(i) $K$ has a unique positive definite matrix-type square root;

(ii) each positive definite kernel $L: X \times X \rightarrow \mathbb{C}$ such that $L \ll K$ has a positive definite matrix-type square root;

(iii) $K \ll c \delta_{X}$ for some constant $c>0$.

(Note that the equivalence of conditions (i)-(iii) above implies that each kernel $L$ appearing in (ii) has in fact a unique pdms root.) We underline here that condition (i) above says about both the existence and the uniqueness of pdms roots. In Sect. 5 we also give equivalent conditions for a positive definite kernel to have at least one such root.

For more information on reproducing kernels consult [4] or [18]. Modern expositions can be found in [5, 21, 22] or [16].

The paper is organized as follows. In Sect. 2 we study in greater detail the collections $\mathscr{V}(A)$ and $\mathscr{Z}(A)$ introduced above, and give a proof of Theorem 1 . Next section contains further conditions (not listed in Theorem 1 ) equivalent to condition (i) of that theorem. The reader can also find there a full description of all possible unitary equivalence types of isometries from $\mathscr{V}(A)$ (see Theorem 6 below). In the fourth part the results of Sect. 2 are applied to unbounded operators. We prove that any truly unbounded positive self-adjoint operator is the absolute value of some positive closed operator that is not self-adjoint (this serves as a criterion for a boundedness of positive self-adjoint operators)—consult Theorem 7. We also show that all closable operators are (in a certain sense) "conditionally" weakly continuous (see Lemma 2 therein). Last, fifth, part is devoted to the notion of a pdms root (for reproducing kernels) introduced above. We gather equivalent conditions for a positive definite kernel to have at least one pdms root (Theorem 8), prove Theorem 2 and study in greater detail kernels having such roots (see, e.g., Theorem 9). In particular, among all such roots (of a fixed kernel) we distinguish one of them which can be seen as (unique) "self-adjoint" (Corollary 4). We also show that if a kernel is a pdms root of some other kernel, then it automatically has a pdms root (item (V) of Theorem 9). This property enables one to define (positive definite matrix-type) roots of higher degrees. Apart from the results of Sect. 4, proofs presented in the last part invoke the machinery of unbounded symmetric operators with Friedrichs' theorem [12] on extending positive operators as the main of them.

\subsection{Notation and terminology}

Throughout this paper all Hilbert spaces are non-trivial and complex, and $H$ denotes one of them. By $\operatorname{dim}(H)$ we denote the Hilbert space dimension of $H$, that is, $\operatorname{dim}(H)$ is the cardinality of an orthonormal basis of $H$. The scalar product of $H$ will be denoted by $\langle\cdot,-\rangle_{H}$. All operators are linear, act between Hilbert spaces and have dense domains. A linear subspace generated by a set $F$ is denoted by $\operatorname{lin}(F)$ and $\varlimsup \operatorname{lin}(F)$ stands for the closure of $\operatorname{lin}(F)$. For any non-empty set $X$ we use $\ell_{2}(X)$ to denote the Hilbert space of all square-summable complex-valued functions on $X$ equipped with the standard inner product. More precisely, $f: X \rightarrow \mathbb{C}$ belongs to 
$\ell_{2}(X)$ if $\sum_{x \in X}|f(x)|^{2}<\infty$; and for $u, v \in \ell_{2}(X),\langle u, v\rangle_{\ell_{2}(X)}=\sum_{x \in X} u(x) \overline{v(x)}$. The canonical basis of $\ell_{2}(X)$ consists of functions $e_{x}$ (where $x$ runs over all elements of $X)$ of the form: $e_{x}(x)=1$ and $e_{x}(y)=0$ for $y \neq x$. For simplicity, we will denote by $\ell_{f i n}(X)$ the linear span of the canonical basis (so, $f: X \rightarrow \mathbb{C}$ belongs to $\ell_{\text {fin }}(X)$ iff the set $\{x \in X: f(x) \neq 0\}$ is finite).

We use $\mathfrak{B}(H)$ and $\mathfrak{H}(H)$ to denote, respectively, the $C^{*}$-algebra of all bounded operators on $H$ and the group of all unitary operators on $H ; I=I_{H}$ is used to denote the unit of $\mathfrak{U}(H)$, and $\mathfrak{B}_{+}(H)$ stands for the collection of all bounded positive operators with trivial kernel. (In particular, each member of $\mathfrak{B}_{+}(H)$ is a selfadjoint operator with dense range.) For two self-adjoint operators $A, B \in \mathfrak{B}(H)$ we write $A \leq B$ or $B \geq A$ if the operator $B-A$ is positive; that is, if $\langle(B-A) x, x\rangle_{H} \geq 0$ for any $x \in H$. By a contraction we mean a bounded operator between Hilbert spaces whose operator norm is not greater than 1 .

Whenever $T$ is an operator, we use $\mathcal{D}(T), \mathcal{N}(T), \mathcal{R}(T)$ and $\Gamma(T)$ to denote, respectively, the domain, the kernel, the range and the graph of $T$. In addition, $\overline{\mathcal{R}}(T)$ denotes the closure of $\mathcal{R}(T)$. The operator $T$ is closed if $\Gamma(T)$ is closed in the product of Hilbert spaces between which $T$ acts. $T$ is closable if the closure of $\Gamma(T)$ is the graph of an operator-in that case $\bar{T}$ denotes the unique operator whose graph coincides with the closure of $\Gamma(T) ; \bar{T}$ is called the closure of $T$ and $\mathcal{D}(T)$ a core of $\bar{T}$. For any closed linear subspace $K$ of $H, P_{K}$ stands for the orthogonal projection from $H$ onto $K$.

Basic facts on the multiplicity theory for bounded self-adjoint operators on separable Hilbert spaces can be found in $\$ 10$ of Chapter IX in [6]. To understand the present paper it is sufficient to know the following result, which will be used several times in this paper: the essential supremum of the multiplicity function of a bounded self-adjoint operator A acting on a separable Hilbert space does not exceed $n \in$ $\{1,2, \ldots\}$ iff $A$ can be decomposed as the direct sum of at most $n$ self-adjoint operators each of which has a cyclic vector. Recall that a vector $v \in H$ is a cyclic vector for a self-adjoint operator $B \in \mathfrak{B}(H)$ if $H=\varlimsup \operatorname{lin}\left(\left\{B^{n} v: n \geq 0\right\}\right)$.

Any closed operator $T: \mathcal{D}(T) \rightarrow K$ (where $\mathcal{D}(T)$ is a dense subspace of $H$ ) admits the so-called polar decomposition which has the form

$$
T=Q A
$$

where $A: \mathcal{D}(T) \rightarrow H$ is positive self-adjoint in $H$ and $Q: H \rightarrow K$ is a partial isometry. Moreover, the above $Q$ and $A$ are uniquely determined by (2) and condition $\mathcal{N}(Q)=\mathcal{N}(T)$. The above operator $A$ satisfies $A^{2}=T^{*} T$ and is called the absolute value of $T$ and denoted by $|T|$. For the details see Theorem 7.20 in [24].

For any $A \in \mathfrak{B}_{+}(H)$ we denote by $\mathscr{V}(A)$ and $\mathscr{Z}(A)$ the collections, respectively, of all isometries $V \in \mathfrak{B}(H)$ such that $A V \in \mathfrak{B}_{+}(H)$, and of all closed linear subspaces $Z$ of $H$ such that $Z \cap \mathcal{R}(A)=\{0\}$. Additionally-for simplicity-for any set $F$ in $H,[F]_{A}$ stands for the set $\overline{\operatorname{lin}}\left(\bigcup_{n=0}^{\infty} A^{n}(F)\right)$; that is, $[F]_{A}$ is the smallest closed linear subspace of $H$ that contains $F$ and is invariant under $A$.

All necessary notions concerning reproducing kernels are introduced and discussed in Sect. 5. 


\section{Isometries of class $\mathscr{V}(\boldsymbol{A})$}

In this section $A$ is fixed and denotes a member of $\mathfrak{B}_{+}(H)$. We begin with

Proposition 1 A function

$$
\Upsilon_{A}: \mathscr{V}(A) \ni V \mapsto \mathcal{R}(V)^{\perp} \in \mathscr{Z}(A)
$$

is a well defined bijection.

Proof First assume that $V \in \mathscr{V}(A)$ and put $Z=\mathcal{R}(V)^{\perp}$. Then $V^{*} A=A V$, hence $\{0\}=\mathcal{N}(A V)=\mathcal{N}\left(V^{*} A\right)=A^{-1}\left(\mathcal{N}\left(V^{*}\right)\right)=A^{-1}(Z \cap \mathcal{R}(A))$, which shows that $Z \cap \mathcal{R}(A)=\{0\}$; that is, $\Upsilon_{A}$ is well defined. Now assume that also $W \in \mathscr{V}(A)$ satisfies $\mathcal{R}(W)^{\perp}=Z$. Define an operator $U \in \mathfrak{U}(H)$ by $U \stackrel{\text { def }}{=} V^{-1} W$ and observe that $W=V U$. It follows from the assumptions that $B \stackrel{\text { def }}{=} A V$ and $C \stackrel{\text { def }}{=} B U$ are bounded positive operators such that $C^{2}=C C^{*}=(B U)\left(U^{*} B\right)=B^{2}$. Since bounded positive operators have unique positive square roots, we infer that $B=C$. Since $B$ has trivial kernel, we get $U=I$ and thus $W=V$. In other words, $\Upsilon_{A}$ is one-to-one.

Now take any $Z \in \mathscr{Z}(A)$ and define $D \in \mathfrak{B}_{+}(H)$ as the (unique) positive square root of $A\left(I-P_{Z}\right) A$. Note that then $D^{2}=\left(\left(I-P_{Z}\right) A\right)^{*}\left(\left(I-P_{Z}\right) A\right)$. It follows that $\mathcal{N}(D)=\mathcal{N}\left(\left(I-P_{Z}\right) A\right)=A^{-1}(Z)=\{0\}$ and hence the range of $D$ is dense in $H$. We also infer that $D=\left|\left(I-P_{Z}\right) A\right|$ and hence-by the properties of the polar decomposition:

$$
\mathcal{R}(D)=\mathcal{R}\left(\left(\left(I-P_{Z}\right) A\right)^{*}\right)=A\left(Z^{\perp}\right) .
$$

(The above formula will be used in the proof of the next result.) Further, since

$$
A^{2}=D^{2}+A P_{Z} A,
$$

we see that $D^{2} \leq A^{2}$ and thus, by [10] (see also Theorem 2.1 in [11]), there is a contraction $V \in \mathfrak{B}(H)$ such that $D=A V$. Observe that then $D=V^{*} A$ and $\mathcal{R}\left(V^{*}\right)$ is dense in $H$. Moreover, it follows from (4) that $A V V^{*} A=A\left(I-P_{Z}\right) A$. Since $A$ has dense range and trivial kernel, we get that $V V^{*}=I-P_{Z}$. Consequently, $V$ is a partial isometry with $\mathcal{R}(V)=\mathcal{R}\left(I-P_{Z}\right)=Z^{\perp}$. But the range of $V^{*}$ is dense in $H$ and hence $V$ is an isometry such that $\mathcal{R}(V)^{\perp}=Z$. A note that $V \in \mathscr{V}(A)$ (because $A V=D$ ) completes the proof.

For any $Z \in \mathscr{Z}(A)$ we use $W_{Z}$ to denote a unique $V \in \mathscr{V}(A)$ such that $\mathcal{R}(V)^{\perp}=$ $Z$ (cf. Proposition 1). Of course, $W_{\{0\}}=I$ is the only unitary operator in $\mathscr{V}(A)$.

Recall that any isometry $V \in \mathfrak{B}(H)$ induces a unique decomposition $H=$ $H_{u} \oplus H_{p}$ (called Wold's decomposition) of the space $H$ such that both $H_{u}$ and $H_{p}$ are invariant under $V, V\left\lceil H_{u}\right.$ is a unitary operator on $H_{u}$ and $\{0\}$ is the only closed linear subspace $K$ of $H_{p}$ such that $V(K)=K$. Each of the spaces $H_{u}$ and $H_{p}$ can be trivial. The restrictions of $V$ to $H_{u}$ and $H_{p}$ are called by us, respectively, the unitary and pure parts of $V$. We call the isometry $V$ pure if $H=H_{p}$. It is well-known (and easy to prove) that $H_{u}=\bigcap_{n=0}^{\infty} \mathcal{R}\left(V^{n}\right)$ and $H_{p}=\bigoplus_{n=0}^{\infty} V^{n}\left(\mathcal{R}(V)^{\perp}\right)$. Any pure isometry $W$ 
is unitarily equivalent to the direct sum of $\alpha$ copies of the (standard) unilateral shift where $\alpha=\operatorname{dim} \mathcal{R}(W)^{\perp}$. The cardinal $\alpha$ defined above is called by us the deficiency index of the isometry $W$. For the proofs of the above facts consult, e.g., Chapter 1 in [17] (therein pure isometries are called shifts and the deficiency index of a pure isometry is called its multiplicity).

Now we describe Wold's decompositions of members of $\mathscr{V}(A)$.

Theorem 3 Let $Z \in \mathscr{Z}(A), V=W_{Z}$ and $H=H_{u} \oplus H_{p}$ be the Wold's decomposition induced by $V$. Then:

- $H_{p}=[Z]_{A}$;

- $H_{u}=\mathcal{N}(V-I)$.

Proof We continue the notation introduced in the proof of Proposition 1: let $D$ denote the positive square root of $A\left(I-P_{Z}\right) A$. Then the formulas (3) and (4) are valid. Moreover, we have $D=A V$ and $\mathcal{N}(D)=0$. Define a bounded operator $T$ : $H \rightarrow H \oplus Z$ by

$$
T x=D x \oplus P_{Z} A x \quad(x \in H) .
$$

We claim that $\mathcal{R}(T)$ is dense in $H \oplus Z$. To convince ourselves of that, fix $u \in H$ and $v \in Z$ such that $u \oplus v \perp \mathcal{R}(T)$. This means that for any $x \in H$,

$$
0=\langle T x, u \oplus v\rangle_{H \oplus Z}=\langle x, D u\rangle_{H}+\langle x, A v\rangle_{H}=\langle x, D u+A v\rangle_{H} .
$$

So, $D u=-A v$, but $D u \in A\left(Z^{\perp}\right)$ (by (3)) and $A v \in A(Z)$. Since $A$ is one-to-one, we get $D u=0=A v$ and therefore $u=v=0$ (as both $A$ and $D$ have trivial kernels).

Further, (5) combined with (4) yields that $\|T x\|^{2}=\|A x\|^{2}$ for any $x \in H$. Since both $T$ and $A$ have dense ranges, we infer that there exists a (unique) unitary operator $Q: H \oplus Z \rightarrow H$ such that

$$
Q T=A \text {. }
$$

Consequently, $T=Q^{-1} A$ and hence (by (5)) $D=P Q^{-1} A$ where $P: H \oplus Z \rightarrow H$ is the projection onto the first coordinate. But $D=V^{*} A$ and $A$ has dense range. So, $V^{*}=P Q^{-1}$. Equivalently, $V=Q P^{*}$. In other words,

$$
V x=Q(x \oplus 0) \quad(x \in H) .
$$

For simplicity, denote by $E$ the subspace $[Z]_{A}$. Since $A^{2}(E) \subset E$ and

$$
\left(A P_{Z} A\right)(E) \subset A(Z) \subset E,
$$

we infer from (4) that $D^{2}(E) \subset E$ and that $D(E) \subset E$ as well.

Now fix $x \in E^{\perp}$. Since $x \perp A^{n}(Z)$, it follows from the self-adjointness of $A$ that $A^{n} x \perp Z$ for any $n \geq 0$. Hence $P_{Z} A^{n} x=0$ and by a simple induction argument applied to (4) we get $A^{2 n} x=D^{2 n} x$ for any $n \geq 0$. Since there is a sequence of polynomials $p_{1}, p_{2}, \ldots$ such that $p_{n}\left(A^{2}\right) \rightarrow A$ and $p_{n}\left(D^{2}\right) \rightarrow D$ in the operator norm as $n \rightarrow \infty$, we obtain $A x=D x$. So, thanks to (7), (5) and (6), 
$V(A x)=V(D x)=Q(D x \oplus 0)=Q(T x)=A x$. But $A\left(E^{\perp}\right) \subset E^{\perp}$ and $A$ is one-toone, thus $A\left(E^{\perp}\right)$ is dense in $E^{\perp}$. We infer that $V z=z$ for all $z \in E^{\perp}$. Since $V$ is an isometry, $V(E) \subset E$. So, to finish the whole proof, it is sufficient to show that $V\lceil E$ is a pure isometry on $E$ (recall that 1 is an eigenvalue of no pure isometry). To this end, we restrict our further considerations to the space $E$ (note that $E$ is invariant for all $A, D$ and $V$ and that $Z \subset E$ and $A\lceil E \cdot V\lceil E=D\lceil E$ ). In other words, we assume that $H=E$. Although everywhere below we will identify $A, V$ and $D$ with their restrictions to $E$, we shall write $E$ instead of $H$ to avoid confusion.

Let

$$
E=E_{u} \oplus E_{p}
$$

be the Wold's decomposition induced by $V$. We only need to show that $E_{p}=E$. To this end, put $U \stackrel{\text { def }}{=} V\left\lceil E_{u} \in \mathfrak{U}\left(E_{u}\right)\right.$ and $S \stackrel{\text { def }}{=} V\left\lceil E_{p} \in \mathfrak{B}\left(E_{p}\right)\right.$ and note that $S$ is a pure isometry on $E_{p}$ and $Z \subset E_{p}$ (as $Z=\mathcal{R}(V)^{\perp}$ ). Represent $A$ as a block matrix $A=$ $\left(\begin{array}{cc}B & X \\ X^{*} & C\end{array}\right)$ with respect to the decomposition (8) (that is, $B: E_{u} \rightarrow E_{u}, X: E_{p} \rightarrow E_{u}$ and $C: E_{p} \rightarrow E_{p}$ ). Then $B \in \mathfrak{B}_{+}\left(E_{u}\right), C \in \mathfrak{B}_{+}\left(E_{p}\right)$ and $D=A V=\left(\begin{array}{cc}B U & X S \\ X^{*} U & C S\end{array}\right)$. Since $D \in \mathfrak{B}_{+}(E)$, we conclude that $B U \in \mathfrak{B}_{+}\left(E_{u}\right)$ and

$$
U X\left(=\left(X^{*} U\right)^{*}\right)=X S .
$$

Then $B^{2}=(B U)(B U)^{*}=(B U)^{2}$. Consequently (by the uniqueness of the positive square root), $B=B U$ and hence $U=I$. So, (9) transforms to $X(I-S)=0$. Since $S$ is a pure isometry, the range of $I-S$ is dense in $E_{p}$. We conclude that $X=0$. So, $A=B \oplus C$ and $E_{p}$ is invariant under $A$. Hence $E=[Z]_{A} \subset E_{p}$ and we are done.

The above result shows that for $Z \in \mathscr{Z}(A)$ the structure of the isometry $W_{Z}$ is completely determined by two cardinal numbers: $\alpha(Z) \stackrel{\operatorname{def}}{=} \operatorname{dim}(Z)$ and $\beta_{A}(Z)=\operatorname{dim}\left([Z]_{A}^{\perp}\right)$. It is a natural question of when it may happen (for a fixed operator $A$ ) that $\beta_{A}(Z)=0$ for some $Z$; that is, when $\mathscr{V}(A)$ contains a pure isometry with a pre-set deficiency index. This question is fully answered in the next three propositions.

Proposition 2 For any $n \in\{1,2, \ldots\}$ the following conditions are equivalent:

(i) there exists a pure isometry $V \in \mathscr{V}(A)$ with deficiency index $n$;

(ii) $H$ is separable, $A$ is non-invertible and the essential supremum of the multiplicity function of $A$ does not exceed $n$;

(iii) $A$ is non-invertible and there is a finite subset $F$ of $H$ such that $[F]_{A}=H$ and $\operatorname{card}(F) \leq n$.

Before giving a proof, let us first separate a special case of the above result that will be applied several times in the sequel: 
Lemma 1 The following conditions are equivalent:

(i) there exists a pure isometry $V \in \mathscr{V}(A)$ with deficiency index 1;

(ii) $H$ is separable, $A$ is non-invertible and has simple spectrum.

Proof If $V \in \mathscr{V}(A)$ is pure and has deficiency index 1 , then $\mathcal{R}(V)^{\perp}$ is generated by a single unit vector, say $z$. In particular, $A$ is non-invertible (since $z \notin \mathcal{R}(A)$ ). Moreover, it follows from Theorem 3 that $H=[z]_{A}$. So, $z$ is a cyclic vector for $A$ and therefore $H$ is separable and $A$ has simple spectrum.

To prove the reverse implication, we model $A$ as the multiplication operator $M_{\mu}$ by independent variable on $L^{2}(\mu)$ where $\mu$ is a probabilistic Borel measure on the spectrum $K \subset[0,\|A\|]$ of $A$ (consult, e.g., Theorem 3.4 in Chapter IX of [6]). That is, $\left(M_{\mu} f\right)(t)=t f(t)$ for any $f \in L^{2}(\mu)$ and $t \in K$. Since $A$ is one-to-one, $\mu(\{0\})=0$. According to Theorem 3, we only need to show that there is $u \notin \mathcal{R}\left(M_{\mu}\right)$ such that $[u]_{M_{\mu}}=L^{2}(\mu)$. Since $A$ (that is, $M_{\mu}$ ) is non-invertible, there is $f \in L^{2}(\mu)$ that is not a value of $M_{\mu}$. This means that $\int_{K} \frac{|f(t)|^{2}}{t^{2}} d \mu(t)=\infty$. By an analogous reasoning, also $u \stackrel{\text { def }}{=} 1+|f| \in L^{2}(\mu)$ is not a value of $M_{\mu}$. It follows from the description of all (closed linear) invariant subspaces of self-adjoint operators of the form $M_{\mu}$ (consult, e.g., Corollary 6.9 in Chapter IX of [6]) that there is a Borel set $\sigma \subset K$ such that $[u]_{M_{\mu}}=\left\{g \in L^{2}(\mu): g=0 \mu\right.$-a.e. on $\left.\sigma\right\}$. But $u \in[u]_{M_{\mu}}$ and therefore $\mu(\sigma)=0$. Consequently, $[u]_{M_{\mu}}=L^{2}(\mu)$ and we are done.

Proof (Proof of Proposition 2) First of all, note that all items (i)-(iii) imply that $H$ is separable and $A$ is non-invertible. So, everywhere below we assume these two properties: that $H$ is separable and $A$ is non-invertible.

Implication "(i) $\Rightarrow\left(\right.$ iii)" is immediate: if $V \in \mathscr{V}(A)$ witnesses (i), then $Z \stackrel{\text { def }}{=} \mathcal{R}(V)^{\perp}$ has an orthonormal basis consisting of $n$ vectors, say $e_{1}, \ldots, e_{n}$. Then $\left[\left\{e_{1}, \ldots, e_{n}\right\}\right]_{A}=[Z]_{A}$ and it follows from Theorem 3 that $[Z]_{A}=H$.

Now assume (iii) holds. Let $F$ be as specified therein. We claim that there are $k \in\{1, \ldots, n\}$ and unit vectors $z_{1}, \ldots, z_{k}$ such that

$$
H=\bigoplus_{j=1}^{k}\left[z_{j}\right]_{A} .
$$

To this end, we proceed by induction on $n$. When $n=1$, our conclusion easily follows. So, assume $n>1$, choose any $a \in F$, put $F_{0} \stackrel{\text { def }}{=} F \backslash\{a\}, H_{0} \stackrel{\text { def }}{=}\left[F_{0}\right]_{A}$ and $A_{0} \stackrel{\text { def }}{=} A\left\lceil H_{0} \in \mathfrak{B}_{+}\left(H_{0}\right)\right.$ and apply the induction hypothesis to $H_{0}$ and $A_{0}$ (and $F_{0}$ ): there are $\ell \in\{1, \ldots, n-1\}$ and unit vectors $z_{1}, \ldots, z_{\ell}$ such that $H_{0}=\bigoplus_{j=1}^{\ell}\left[z_{j}\right]_{A_{0}}$. If $H_{0}=H$, just put $k=\ell$ to finish the proof of (10). When $H_{0} \neq H$, proceed as follows. Since $H=[F]_{A}$ coincides with the closure of $[a]_{A}+\left[F_{0}\right]_{A}=[a]_{A}+H_{0}$, the subspace $P_{H_{0}^{\perp}}\left([a]_{A}\right)$ is dense in $H_{0}^{\perp}$. Further, $P_{H_{0}^{\perp}}$ commutes with $A$ and thus $P_{H_{0}^{\perp}}\left([a]_{A}\right)$ is dense in $\left[P_{H_{0}^{\perp}}(a)\right]_{A}$. So, $\left[P_{H_{0}^{\perp}}(a)\right]_{A}=H_{0}^{\perp}, b \stackrel{\text { def }}{=} P_{H_{0}^{\perp}}(a) \neq 0$ and it is sufficient to define $k$ as $\ell+1$ and $z_{k}$ as $\frac{b}{\|b\|}$ to get (10). Since each of the subspaces 
$\left[z_{j}\right]_{A}$ is invariant under $A$ (and $z_{j}$ is a cyclic vector for the restriction of $A$ to $\left[z_{j}\right]_{A}$ ), we see that $A$ is the direct sum of at most $n$ self-adjoint operators with simple spectrum-which yields (ii).

Finally, assume that (ii) is fulfilled. This means that $A$ is the direct sum of at most $n$ self-adjoint operators with simple spectrum, say $A=\bigoplus_{j=1}^{k} A_{j}$ where $k \leq n$ (and $A_{j}$ has simple spectrum). Then each of $A_{j}$ is positive with trivial kernel and one of them, say $A_{1}$, is non-invertible. Using e.g. the spectral measure of $A_{1}$, we can decompose $A_{1}$ as $A_{1}=\bigoplus_{m=1}^{\infty} B_{m}$ where each $B_{m}$ acts on a non-zero Hilbert space and $\lim _{m \rightarrow \infty}\left\|B_{m}\right\|=0$. Now we decompose the set of all positive integers as the union of $n$ pairwise disjoint sets $J_{1}, \ldots, J_{n}$ in a way such that $J_{1}$ is infinite and for any $j \in\{2, \ldots, n\}$ :

- if $A_{j}$ is non-invertible, then $J_{j}=\varnothing$;

- if $A_{j}$ is invertible, then $J_{j}$ is infinite and $\left\|B_{s}\right\|<1 /\left\|A_{j}^{-1}\right\|$ for each $s \in J_{j}$.

Now define operators $C_{1}, \ldots, C_{n}$ as follows:

- $C_{1}=\bigoplus_{s \in J_{1}} B_{s}$;

- $C_{j}=A_{j}$ if $j>1$ and $A_{j}$ is non-invertible;

- $C_{j}=\left(\bigoplus_{s \in J_{j}} B_{s}\right) \oplus A_{j}$ in all other cases.

It follows from the above construction that:

- $A$ is unitarily equivalent to $\bigoplus_{j=1}^{n} C_{j}$;

- each of $C_{j}$ is positive, non-invertible and has trivial kernel;

- each of $C_{j}$ has simple spectrum.

Only the last of these properties can be seen as non-trivial, so let us briefly explain it. Since $B_{j} \stackrel{\text { def }}{=} \bigoplus_{s \in J_{j}} B_{s}$ is the restriction of $A_{1}$ to an invariant subspace of $A_{1}$ and $A_{1}$ has simple spectrum, $B_{j}$ has simple spectrum as well. Finally, if $j>1$ and $A_{j}$ is invertible, then $\left\|B_{j}\right\|<1 /\left\|A_{j}^{-1}\right\|$ which implies that the spectra of $B_{j}$ and $A_{j}$ are disjoint. But then $B_{j} \oplus A_{j}$ has simple spectrum, as both $A_{j}$ and $B_{j}$ have so.

To conclude the proof, apply Lemma 1 to each of $C_{j}$ : there is a pure isometry $S_{j}$ with deficiency index 1 (acting on an appropriate Hilbert space) such that $C_{j} S_{j}$ is positive. Then also $\left(\bigoplus_{j=1}^{n} C_{j}\right)\left(\bigoplus_{j=1}^{n} S_{j}\right)$ is positive. So, we complete the proof by noticing that $\bigoplus_{j=1}^{n} S_{j}$ is a pure isometry with deficiency index $n$ and that $A$ is unitarily equivalent to $\bigoplus_{j=1}^{n} C_{j}$.

Proposition 3 The following conditions are equivalent:

(i) there exists a pure isometry $V \in \mathscr{V}(A)$ with deficiency index $\aleph_{0}$;

(ii) $H$ is separable and $A$ is non-invertible.

Proof The argument is similar to a part of the previous proof and goes as follows. It is clear that (ii) is implied by (i). Assume (i) holds and let $B$ be a maximal set of unit 
vectors in $H$ such that $B \backslash \mathcal{R}(A) \neq \varnothing$ and $[b]_{A} \perp[c]_{A}$ for distinct $b, c \in B$. Then $B$ is non-empty and (at most) countable, and

$$
H=\bigoplus_{b \in B}[b]_{A} .
$$

We fix $e \in B$ such that $e \notin \mathcal{R}(A)$. We infer that $A_{0} \stackrel{\text { def }}{=} A\left\lceil[e]_{A}\right.$ is non-invertible (as an operator in $\mathfrak{B}\left([e]_{A}\right)$ ). So, we may decompose $A_{0}$ (using, e.g., the spectral measure of $\left.A_{0}\right)$ as $A_{0}=\bigoplus_{n=1}^{\infty} D_{n}$ where each $D_{n}$ acts on a non-zero Hilbert space and $\lim _{n \rightarrow \infty}\left\|D_{n}\right\|=0$. Denote by $C$ the set of all $b \in B$ such that $A\left\lceil[b]_{A}\right.$ is invertible (in $\left.\mathfrak{B}\left([b]_{A}\right)\right)$. We divide the set of all positive integers into pairwise disjoint sets $J_{b}(b \in$ $B)$ in a way such that:

- $J_{e}$ is infinite;

- if $b \in C$, then $J_{b}$ is infinite and $\left\|D_{s}\right\|<1 / \|\left(A\left\lceil[b]_{A}\right)^{-1} \|\right.$ for all $s \in J_{b}$;

- $J_{b}=\varnothing$ in all other cases.

Finally, decompose $J_{e}$ as the union of pairwise disjoint infinite sets $I_{0}, I_{1}, \ldots$ Now we define operators $T_{b}(b \in B)$ and $T^{(n)}(n=1,2, \ldots)$ by the rules:

- $T_{e}=\bigoplus_{k \in I_{0}} D_{k}$;

- $T_{b}=\left(\oplus_{s \in J_{b}} D_{s}\right) \oplus\left(A\left\lceil[b]_{A}\right)\right.$ for all $b \in C$;

- $T_{b}=A\left\lceil[b]_{A}\right.$ for all $b \in B \backslash(C \cup\{e\})$;

- $T^{(n)}=\bigoplus_{k \in I_{n}} D_{k}$ for all $n>0$.

For simplicity, gather all the operators defined above in a sequence $L_{1}, L_{2}, \ldots$ Since all the sets $I_{n}(n \geq 0)$ and $J_{b}(b \in B \backslash\{e\})$ are pairwise disjoint and their union coincides with the set of all positive integers, one shows that $A$ is unitarily equivalent to $\bigoplus_{n=1}^{\infty} L_{n}$ (thanks to (11)). Furthermore, it follows from the construction that each of $L_{n}$ is non-invertible and has a cyclic vector (cf. the proof of Proposition 2). So, thanks to Lemma 1 for any $n>0$ there is a pure isometry $S_{n}$ with deficiency index 1 (that acts on a suitable Hilbert space) such that $L_{n} S_{n}$ is positive. Then $S \stackrel{\text { def }}{=} \bigoplus_{n=1}^{\infty} S_{n}$ is a pure isometry with deficiency index $\aleph_{0}$ and belongs to $\mathscr{V}\left(\bigoplus_{n=1}^{\infty} L_{n}\right)$. This easily implies that (i) holds.

Proposition 4 Let $\alpha$ be an uncountable cardinal number and E denote the spectral measure of $A$. The following conditions are equivalent:

(i) there exists a pure isometry $V \in \mathscr{V}(A)$ with deficiency index $\alpha$;

(ii) $\operatorname{dim}(H)=\alpha$ and there is $Z \in \mathscr{Z}(A)$ such that $\operatorname{dim}(Z)=\alpha$;

(iii) $\operatorname{dim}(H)=\alpha$ and $\operatorname{dim} \mathcal{R}(E((0, \varepsilon)))=\alpha$ for any $\varepsilon>0$.

Proof First of all, note that all conditions specified in the proposition imply that

$$
\operatorname{dim}(H)=\alpha .
$$

Thus, everywhere below we assume (12). Note also that (ii) is implied by (i) thanks to Proposition 1. 
Now assume (ii) holds and let $Z$ be as specified therein. Let $B$ be a maximal set of unit vectors in $Z$ such that $[b]_{A} \perp[c]_{A}$ for all distinct $b, c \in B$. We claim that

$$
\operatorname{card}(B)=\alpha .
$$

To convince oneself of that, assume $\operatorname{card}(B)<\alpha$. Then also $\operatorname{dim}\left(\bigoplus_{b \in B}[b]_{A}\right)<\operatorname{dim}(Z)$ and therefore there exists a unit vector $z \in Z$ orthogonal to $\bigoplus_{b \in B}[b]_{A}$. This yields that $[b]_{A} \perp[z]_{A}$ for any $b \in B$ which contradicts the maximality of $B$. So, (13) holds.

Now for each $b \in B$ denote by $P_{b}$ the orthogonal projection onto $[b]_{A}$. Additionally, for a fixed $\varepsilon>0$ set $Q \stackrel{\text { def }}{=} E((0, \varepsilon))$. Note that $Q$ commutes with all $P_{b}$ and that to get (iii) it is sufficient to show that $Q P_{b} \neq 0$ for any $b \in B$. To this end, fix $b \in B$ and assume that, on the contrary, $Q P_{b}=0$. Then $[b]_{A} \subset E([\varepsilon, \infty))$. This means that $\langle A x, x\rangle_{H} \geq \varepsilon\|x\|^{2}$ for any $x \in[b]_{A}$. Consequently, $A\left\lceil[b]_{A}\right.$ is invertible. But $[b]_{A}$ is invariant for $A$ and $b \notin \mathcal{R}(A)$ which contradicts the invertibility of that restriction.

Finally, assume (iii) holds. We want to show that there is $V$ witnessing (i). Let $\left\{E_{s}\right\}_{s \in S}$ be a maximal family of closed linear subspaces such that:

- $E_{s}$ is separable and $A\left(E_{s}\right) \varsubsetneqq E_{s}$ for all $s \in S$;

- $E_{s} \perp E_{t}$ for any distinct $s, t \in S$.

We claim that

$$
\operatorname{card}(S)=\alpha
$$

To see this, set

$$
F \stackrel{\text { def }}{=}\left(\bigoplus_{s \in S} E_{s}\right)^{\perp}
$$

and note that $A(F) \subset F$. Actually, we have $A(F)=F$. Indeed, if there was $z \in F \backslash A(F)$, then $[z]_{A}$ would be separable and orthogonal to all $E_{S}$ which would contradict the maximality of the family $\left\{E_{s}\right\}_{s \in S}$.

Further, it follows from the inverse mapping theorem that for some $\varepsilon>0$, $\langle A x, x\rangle_{H} \geq \varepsilon\|x\|^{2}$ for all $x \in F$. This inequality implies that $E((0, \varepsilon)) P_{F}=0$. Consequently, $\mathcal{R}(E((0, \varepsilon))) \subset F^{\perp}=\bigoplus_{s \in S} E_{s}$. So, the conclusion of (iii) and the separability of all $E_{s}$ yields (14).

Further, keeping the setting (15), decompose $F$ as $F=\bigoplus_{t \in T} W_{t}$ where each $W_{t}$ is a separable closed linear subspace of $F$ invariant under $A$. Then $\operatorname{card}(T) \leq \alpha$ and hence there is a one-to-one mapping $\kappa: T \rightarrow S$. Now define, for $s \in S, H_{s}$ as follows:

- $H_{s}=E_{s}$ provided $s \notin \kappa(T)$;

- $H_{s}=E_{s} \oplus W_{\kappa^{-1}(s)}$ otherwise.

Observe that $H=\bigoplus_{s \in S} H_{s}$, and for any $s \in S, H_{s}$ is separable and $A\left(H_{s}\right) \varsubsetneqq H_{s}$. So, it follows from Proposition 3 that for any $s \in S$ there is a pure isometry $V_{s}: H_{s} \rightarrow H_{s}$ 
that belongs to $\mathscr{V}\left(A\left\lceil H_{s}\right)\right.$. To complete the proof, define $V \in \mathscr{V}(A)$ by $V=\bigoplus_{s \in S} V_{s}$ and note that $V$ is a pure isometry with deficiency index $\alpha$ (by (14)).

Observe that item (ii) of the above theorem-as well as the collection $\mathscr{Z}(A)$ depends only on the range of the operator $A$. Further conditions (also formulated only in terms of operator ranges) equivalent to this item are a subject of Theorem 5 from the next section.

Proof (Proof of Theorem 1) Just observe that the equivalence of conditions (i) and (ii) immediately follows from Propositions 2, 3 and 4, whereas the remaining (additional) part of the theorem is a reformulation of Lemma 1.

Remark 1 It was shown earlier-in [13] - that one-to-one Hankel operators satisfying the double positivity condition have simple spectra. Both the proofs-in the paper cited above and ours-are based on the same idea, which is a kind of folklore in operator theory. For the details, consult [14] or Proposition 2.5 in [13] together with the preceding paragraph (therein).

The following result is a simple consequence of a deep theorem from [15]. Here we give its brief proof.

Corollary 1 The essential supremum of the multiplicity function of a positive Hankel operator A with trivial kernel does not exceed 2.

Proof If $S$ denotes the (classical) unilateral shift, then $S^{2}$ is a pure isometry with deficiency index 2 and $A S^{2}$ is a positive operator (because $A$ is positive and Hankel). So, the assertion follows from Theorem 1 (for $m=2$ ).

In [13] the authors showed that for any bounded Hankel operator $A$ satisfying the double positivity condition the operator $A\lceil\overline{\mathcal{R}}(A)$ has simple spectrum. It is also well-known (and easy to prove) that the kernel of a Hankel operator is either trivial or infinite-dimensional. So, the following problem naturally arises:

Conjecture 1 Let A be a bounded positive operator on a separable Hilbert space such that $\mathcal{N}(A)$ is infinite-dimensional, $\mathcal{R}(A)$ is non-closed and $A\lceil\overline{\mathcal{R}}(A)$ has simple spectrum. Then A is unitarily equivalent to a Hankel operator satisfying the double positivity condition.

\section{Operator ranges}

In this part we give further conditions on a dense operator range $\mathcal{R}$ contained in $H$ equivalent to the existence of a closed linear subspace $Z$ of $H$ such that $Z \cap \mathcal{R}=$ $\{0\}$ and $\operatorname{dim}(Z)=\operatorname{dim}(H)$ (cf. item (ii) in Proposition 4). To this end we need to recall some well-known facts about operator ranges.

A linear subspace $\mathcal{R}$ of a Hilbert space $H$ is called an operator range if there exist a Hilbert space $K$ and a bounded operator $T: K \rightarrow H$ such that $\mathcal{R}(T)=\mathcal{R}$. The following is a basic result on operator ranges (see Theorem 1.1 in [11]). 
Theorem 4 For any operator range $\mathcal{R}$ in $H$ there are mutually orthogonal closed linear subspaces $\mathrm{H}_{1}, \mathrm{H}_{2}, \ldots$ such that

$$
\mathcal{R}=\operatorname{ran}\left[H_{1}, H_{2}, \ldots\right] \stackrel{\text { def }}{=}\left\{\oplus_{n=1}^{\infty} x_{n} \in \bigoplus_{n=1}^{\infty} H_{n}: \sum_{n=1}^{\infty}\left(2^{n}\left\|x_{n}\right\|\right)^{2}<\infty\right\} .
$$

Moreover, the operator range $\mathcal{R}$ given by (16) is dense in $H$ iff $H=\bigoplus_{n=1}^{\infty} H_{n}$.

It is worth noting that for a given operator range $\mathcal{R}$ a sequence $H_{1}, H_{2}, \ldots$ such that $\mathcal{R}=\operatorname{ran}\left[H_{1}, H_{2}, \ldots\right]$ (cf. (16)) is, in general, not unique. Also, some of the spaces $H_{n}$ can be zero-dimensional. For the purposes of this section, let us introduce the following

Definition 2 Let $\alpha_{1}, \alpha_{2}, \ldots$ be any sequence of cardinal numbers. We say an operator range $\mathcal{R}$ in $H$ is of type $\mathrm{R}\left(\alpha_{n}\right)_{n=1}^{\infty}$ if there are mutually orthogonal closed linear subspaces $H_{1}, H_{2}, \ldots$ of $H$ such that $\mathcal{R}=\operatorname{ran}\left[H_{1}, H_{2}, \ldots\right]$ (cf. (16)) and $\operatorname{dim}\left(H_{n}\right)=\alpha_{n}$ for any $n>0$.

Two operator ranges $\mathcal{R}_{1}$ in $H_{1}$ and $\mathcal{R}_{2}$ in $H_{2}$ are equivalent if there is a unitary operator $U: H_{1} \rightarrow H_{2}$ such that $U\left(\mathcal{R}_{1}\right)=\mathcal{R}_{2}$.

A single operator range can be of many different types. However, it is easy to see that dense operator ranges of a given type are all equivalent. When two types represent equivalent dense operator ranges is a subject of Theorem 3.3 in [11]. Here we skip the details.

The main result of this section is

Theorem 5 Let $\mathcal{R}$ be a dense operator range of type $\mathrm{R}\left(\alpha_{n}\right)_{n=1}^{\infty}$ in a Hilbert space $H$ of dimension $\alpha \geq \aleph_{0}$. The following conditions are equivalent:

(a) there exists a closed linear subspace $Z$ of $H$ such that $Z \cap \mathcal{R}=\{0\}$ and $\operatorname{dim}(Z)=\alpha$;

(b) for any $\beta<\alpha$ there exists a closed linear subspace $Y$ of $H$ such that $Y \cap \mathcal{R}=$ $\{0\}$ and $\operatorname{dim}(Y)>\beta$;

(c) for any $\beta<\alpha$ and $n>0$ there is $m>n$ such that $\sum_{j=n+1}^{m} \alpha_{j}>\beta$;

(d) $\operatorname{dim}\left(K^{\perp}\right)=\alpha$ for any linear subspace $K$ of $\mathcal{R}$ that is closed in $H$;

(e) there exists a dense operator range $\mathcal{R}^{\prime}$ in $H$ such that $\mathcal{R}^{\prime} \cap \mathcal{R}=\{0\}$;

(f) there exists $U \in \mathfrak{U}(H)$ for which $U(\mathcal{R}) \cap \mathcal{R}=\{0\}$;

(g) there exists a closed linear subspace $W$ of $H$ such that $W \cap \mathcal{R}=W^{\perp} \cap \mathcal{R}=\{0\}$.

Moreover, $\operatorname{dim}(W)=\operatorname{dim}\left(W^{\perp}\right)=\alpha$ for any $W$ witnessing $(\mathrm{g})$.

Before giving a proof, first we comment on the assertion of (f) in the above result: in the case when $\mathcal{R}$ is a non-closed operator range in a separable Hilbert space $H$ it was first proved by Dixmier [8] (consult also Theorem 3.6 in [11]). He started his proof with a specific example of an operator range for which (f) holds and then described an elegant technique to get the assertion in full generality (in the separable case). Here we will generalize his method, but instead of using his "starting" 
example we propose a new approach to this issue-our starting tool will be the following proposition, which may be already known, but we could not find it in the literature. This result can be considered interesting in its own right.

Proposition 5 Let $C: K \rightarrow H$ be a compact operator from a Hilbert space $K$ into a separable infinite-dimensional Hilbert space $H$. Then the set $\Delta$ of all $U \in \mathfrak{U}(H)$ such that $U(\mathcal{R}(C)) \cap \mathcal{R}(C)=\{0\}$ is dense $\mathscr{G}_{\delta}$ in the Polish group $\mathfrak{U}(H)$ equipped with the strong operator topology.

Proof First of all, recall that for separable $H$ the space $\mathfrak{U}(H)$ is separable and completely metrizable in the strong operator topology. Moreover, for any Hilbert space $K, \mathfrak{U}(K)$ is a topological group with respect to this topology. So, in the separable case we can apply Baire's theorem, which will lead us to the final conclusion after showing the following property, valid in all (that is, possibly nonseparable) infinite-dimensional Hilbert spaces $H$ :

( $\star$ For any norm compact set $M \subset H$ disjoint from the origin, the set $\Omega$ of all $U \in \mathfrak{U}(H)$ such that $U(M) \cap M=\varnothing$ is open and dense in the topological group $\mathfrak{U}(H)$ equipped with the strong operator topology.

First we prove $(\star)$, then we will show how it leads to the whole conclusion of the proposition.

We may and do assume that $M \neq \varnothing$. It easily follows from the compactness of $M$ that $\Omega$ is open in $\mathfrak{U}(H)$. Indeed, fix $U \in \Omega$ and take $\delta>0$ such that $\| U x-$ $y \| \geq 4 \delta$ for all $x, y \in M$. Further, let $F \subset M$ be a finite non-empty $\delta$-net in $M$. It is then easy to check that $\|V x-y\| \geq \delta$ for all $x, y \in M$ and any $V \in \mathfrak{U}(H)$ such that $\|V f-U f\|<\delta$ for any $f \in F$. Consequently, each such $V$ belongs to $\Omega$ and hence $\Omega$ is open in the strong operator topology. To show that $\Omega$ is dense in $\mathfrak{U}(H)$, we need to know that $H$ is infinite-dimensional. Fix $U \in \mathfrak{U}(H)$, a finite non-empty set $F$ in $H$ and $\varepsilon>0$. Our aim is to show that there is $V \in \Omega$ such that $\|V f-U f\| \leq \varepsilon$ for any $f \in F$. We may and do assume that $0 \notin F$. For simplicity, put $L \stackrel{\text { def }}{=} M \cup F$ and take $m>0$ such that

$$
\|y\| \geq m \quad(y \in L) .
$$

Next, choose any $\delta \in(0,1)$ satisfying

$$
2\|f\| \delta<\varepsilon \quad(f \in F)
$$

and let $S \subset L$ be a finite $(m \delta / 4)$-net in $L$ containing $F$. Set $E \stackrel{\text { def }}{=} \operatorname{lin}(S)$ and take any $W \in \mathfrak{U}(H)$ such that

$$
W(E) \perp U(E)+E .
$$

We conclude from the above property (and the fact that $E$ is finite-dimensional) that there exists $V \in \mathfrak{U}(H)$ that satisfies

$$
V x=\delta W x+\sqrt{1-\delta^{2}} U x \quad(x \in E) .
$$


Since $F \subset E$, it follows from (19) and (20) that for any $f \in F$,

$$
\|V f-U f\|^{2}=2\left(1-\sqrt{1-\delta^{2}}\right)\|f\|^{2} \leq 2 \delta^{2}\|f\|^{2}<\varepsilon^{2}
$$

(where the last inequality is a consequence of (18)). Thus, to end the proof of $(\star)$, it remains to check that $V(M) \cap M=\varnothing$. To this end, first take $a, b \in S \subset E \cap L$. Again, we infer from (19) and (20) that $\|V a-b\|^{2}=\|a\|^{2}+\|b\|^{2}-2 \sqrt{1-\delta^{2}} \operatorname{Re}\langle U a, b\rangle_{H}$, so

$$
\|V a-b\|^{2} \geq\|a\|^{2}+\|b\|^{2}-2 \sqrt{1-\delta^{2}}\|a\| \cdot\|b\| \geq \delta^{2}\|b\|^{2} \geq \delta^{2} m^{2},
$$

by (17). Now if $x, y \in M$ are arbitrary, choose $a, b \in S$ such that $\|x-a\| \leq m \delta / 4$ and $\|y-b\| \leq m \delta / 4$. We then have $\|V x-y\| \geq\|V a-b\|-\|V a-V x\|-\| y-$ $b \| \geq m \delta / 2$ and we are done.

Having $(\star)$, the assertion of the proposition can briefly be proven. Since $C$ is compact and the closed unit ball $\bar{B}_{K}$ in $K$ is weakly compact, the set $D \stackrel{\text { def }}{=} C\left(\bar{B}_{K}\right)$ is norm compact. Observe that $\mathcal{R}(C) \backslash\{0\}=\bigcup_{n=1}^{\infty} M_{n}$ where $M_{n}=n C \backslash\left(\frac{1}{n} B_{H}\right)$ where $B_{H}$ is the open unit ball in $H$. We infer from $(\star)$ that the set $\Omega_{n} \stackrel{\text { def }}{=}\{U \in$ $\left.\mathfrak{U}(H): U\left(M_{n}\right) \cap M_{n}=\varnothing\right\}$ is open and dense in $\mathfrak{U}(H)$. Finally, Baire's theorem yields that the intersection of all $\Omega_{n}$, which coincides with $\Delta$, is dense in $\mathfrak{U}(H)$.

In the proof of Theorem 5 we shall also apply the following result.

Corollary 2 There exists a dense operator range $\mathcal{R}_{0}$ in a separable Hilbert space $K$ and a closed linear subspace $W$ of $K$ such that $W \cap \mathcal{R}_{0}=W^{\perp} \cap \mathcal{R}_{0}=\{0\}$.

Proof Let $K \stackrel{\text { def }}{=} H \oplus H$ (where $H$ is infinite-dimensional and separable) and $W \stackrel{\text { def }}{=} H \oplus\{0\} \subset K$. Take any compact self-adjoint operator $A: H \rightarrow H$ with trivial kernel and choose-applying Proposition 5-any $U \in \mathfrak{U}(H)$ such that

$$
U(\mathcal{R}(A)) \cap \mathcal{R}(A)=\{0\} .
$$

Let $B: H \rightarrow K$ be given by $B x \stackrel{\text { def }}{=} A x \oplus U A U^{-1} x$ and set $\mathcal{R}_{0} \stackrel{\text { def }}{=} \mathcal{R}(B)$. Since both $A$ and $U$ are one-to-one, we have $W \cap \mathcal{R}_{0}=W^{\perp} \cap \mathcal{R}_{0}=\{0\}$. So, we only need to show that $\mathcal{R}_{0}$ is dense in $K$, which simply follows from (21): if $a \oplus b$ is orthogonal to $\mathcal{R}_{0}$, then $A a+U A U^{-1} b=0$ and, consequently, $a=b=0$.

Proof (Proof of Theorem 5) For the aim of this proof, take a sequence of mutually orthogonal closed linear subspaces $H_{1}, H_{2}, \ldots$ of $H$ such that

$$
\mathcal{R}=\operatorname{ran}\left[H_{1}, H_{2}, \ldots\right]
$$

and $\operatorname{dim}\left(H_{n}\right)=\alpha_{n}$ for any $n>0$. Since $\mathcal{R}$ is dense, we have (by Theorem 4):

$$
H=\bigoplus_{n=1}^{\infty} H_{n} \text {. }
$$

Before passing to the main proof, consider an additional condition: 
1 for any $\beta<\alpha$ and $n>0$ there exists a closed linear subspace $K$ of $H$ such that

$$
K \cap\left(\bigoplus_{j=1}^{n} H_{j}\right)=\{0\} \quad \text { and } \quad \operatorname{dim}(K)>\beta .
$$

(Note that (h) is a weakening of each of (a), (b), (c), (d) and (g).) We will show that (h) is equivalent to each of (a)-(g). For the reader's convenience, let us draw the scheme of the proof: $(\mathrm{a}) \Rightarrow(\mathrm{b}) \Rightarrow(\mathrm{d}) \Rightarrow(\mathrm{h}) \Rightarrow(\mathrm{c}) \Rightarrow(\mathrm{a})$; then $(\mathrm{f}) \Rightarrow(\mathrm{e}) \Rightarrow(\mathrm{h})$ and $(\mathrm{g}) \Rightarrow(\mathrm{a})$ (together with the additional claim of the theorem), and finally (c) $\Rightarrow(\mathrm{f}),(\mathrm{g})$.

Of course, (a) implies (b). If $\beta<\alpha$ and $Y$ witnesses (b) (for $\beta$ ), and $K$ is as specified in (d), then $P_{K^{\perp}}$ is one-to-one on $Y$. Consequently, $\operatorname{dim}\left(K^{\perp}\right)=$ $\operatorname{dim}\left(\mathcal{R}\left(P_{K^{\perp}}\right)\right) \geq \operatorname{dim}(Y)>\beta$ which yields (d). It is obvious that (d) implies (h). Now assume (h) holds and fix $\beta<\alpha$ and $n \geq 0$. For simplicity, denote by $P$ the orthogonal projection onto the orthogonal complement (in $H$ ) of $\bigoplus_{j=1}^{n} H_{j}$. By (h), there is a closed linear subspace $K$ of $H$ that satisfies (24). As argued previously, we conclude that $\operatorname{dim}(\mathcal{R}(P)) \geq \operatorname{dim}(K)>\beta$. But $\mathcal{R}(P)=\bigoplus_{j=n+1}^{\infty} H_{j}$ (thanks to (23)) and hence $\sum_{j>n} \alpha_{j}>\beta$. So, one can find $m>n$ such that $\sum_{j=n+1}^{m} \alpha_{j}>\beta$ which gives (c). Finally, assume (c) holds. Consider a bounded operator $A: H \rightarrow H$ defined as follows:

$$
A\left(\oplus_{n=1}^{\infty} x_{n}\right) \stackrel{\text { def }}{=} \oplus_{n=1}^{\infty} 2^{-n} x_{n}, \quad \oplus_{n=1}^{\infty} x_{n} \in \bigoplus_{n=1}^{\infty} H_{n} .
$$

It readily follows from (22) that $\mathcal{R}(A)=\mathcal{R}$. Moreover, it is also easy to show that for arbitrarily fixed $\varepsilon>0, \bigoplus_{n=j}^{\infty} H_{j} \subset \mathcal{R}(E((0, \varepsilon)))$ for sufficiently large $n>0$ where $E$ is the spectral measure of $A$. Consequently, we infer from (c) that $\operatorname{dim}(\mathcal{R}(E((0, \varepsilon))))=\alpha$ and it suffices to apply Proposition 4 to get (a).

Further, (e) is easily implied by (f) as $U(\mathcal{R})$ (for any $U \in \mathfrak{U}(H)$ ) is a dense operator range in $H$. And if (e) holds, $\mathcal{R}^{\prime}=\operatorname{ran}\left[H_{1}^{\prime}, H_{2}^{\prime}, \ldots\right]$ for suitable sequence $H_{1}^{\prime}, H_{2}^{\prime}, \ldots$ of mutually orthogonal closed linear subspaces of $H$ such that $H=\bigoplus_{n=1}^{\infty} H_{n}^{\prime}$. Then $\sum_{n=1}^{\infty} \operatorname{dim}\left(H_{n}^{\prime}\right)=\alpha$ and $\left(\bigoplus_{j=1}^{m} H_{j}^{\prime}\right) \cap\left(\bigoplus_{k=1}^{n} H_{k}\right)=\{0\}$ for all $n, m>0$. These properties easily yield (h).

To show the additional claim of the theorem and that (a) follows from (g), it is sufficient to prove that $\operatorname{dim}(W)=\alpha$ whenever $W$ is as specified in (g). To this end, assume - on the contrary - that $\operatorname{dim}(W)<\alpha$. Then, by (23), we can find $n>0$ such that $\sum_{j=1}^{n} \operatorname{dim}\left(H_{j}\right)>\operatorname{dim}(W)$. This inequality implies that $\left(\bigoplus_{j=1}^{n} H_{j}\right) \cap W^{\perp} \neq\{0\}$ which contradicts $(\mathrm{g})$ as $\bigoplus_{j=1}^{n} H_{j} \subset \mathcal{R}$.

We turn to the hardest part of the proof-namely, that both (f) and (g) follow from (c). We adapt Dixmier's proof [8] (see also Theorem 3.6 in [11]) of the result mentioned in the paragraph following the statement of Theorem 5 above, but instead of his specific example of a dense operator range in a separable Hilbert space that satisfies (f) we apply our Proposition 5 and Corollary 2.

To simplify further arguments, let us call a linear subspace $\mathcal{R} \subset H$ of an arbitrary Hilbert space $(\mathrm{f}, \mathrm{g})$-valid if both $(\mathrm{f})$ and $(\mathrm{g})$ hold for $\mathcal{R}$. Here we do not assume that $\mathcal{R}$ is an operator range. In a similar manner we define (f)-valid and (g)-valid linear subspaces of Hilbert spaces. Moreover, for any non-empty set $J$ and $\mathcal{R} \subset H$ let 
$\mathcal{R}^{J} \subset \bigoplus_{j \in J} H_{j}$ (with $H_{j}=H$ for all $j \in J$ ) stand for the set of all $\oplus_{j \in J} x_{j}$ with $x_{j} \in \mathcal{R}$ for any $j \in J$. Finally, for any infinite cardinal $\gamma$ we call an operator range $\mathcal{R}$ of type $\mathrm{R}_{\gamma}$ if it is of type $\mathrm{R}\left(\gamma_{n}\right)_{n=1}^{\infty}$ where $\gamma_{n}=\gamma$ for each $n$. We divide the remaining part of the proof of the theorem into the following steps:

(I) There are dense operator ranges $\mathcal{R}_{f}$ and $\mathcal{R}_{g}$ in separable Hilbert spaces such that $\mathcal{R}_{f}$ is (f)-valid and $\mathcal{R}_{g}$ is (g)-valid.

(II) If $\mathcal{R}$ is (f,g)-valid, each linear subspace of $\mathcal{R}$ is (f,g)-valid as well.

(III) If $\mathcal{R}$ is (f)-valid (resp. (g)-valid), so is $\mathcal{R}^{J}$ for any set $J \neq \varnothing$.

(IV) If $\mathcal{R}$ is a dense operator range in a separable Hilbert space $H$ and $J$ is an infinite set, then $\mathcal{R}^{J}$ contains an operator range of type $\mathrm{R}_{\text {card }(J)}$ that is dense in $H^{J}$.

(V) For any infinite cardinal $\gamma$, all dense operator ranges of type $\mathbf{R}_{\gamma}$ are (f,g)valid.

(VI) If $\mathcal{R}$ and $H$ are as specified in the statement of the theorem and (c) is fulfilled, then $\mathcal{R}$ is contained in a dense (in $H$ ) operator range of type $\mathrm{R}_{\alpha}$. In particular, $\mathcal{R}$ is (f,g)-valid.

Note that property (VI) is exactly what we want. Below we give brief proofs of the above items (I)-(VI).

Property (I) is covered by Proposition 5 and Corollary 2; (II) is obvious; whereas (III) follows from a simple argument on direct sums: if $U$ is a unitary operator on $H$ such that $U(\mathcal{R}) \cap \mathcal{R}=\{0\}$, then $U^{J} \stackrel{\text { def }}{=} \bigoplus_{j \in J} U_{j}$ (with $U_{j}=U$ for all $j \in J$ ) is a unitary operator on $H^{J}$ such that $U^{J}\left(\mathcal{R}^{J}\right) \cap \mathcal{R}^{J}=\varnothing$ (similarly: if $W \subset H$ witnesses (g) for $\mathcal{R}$, then $W^{J}$ witnesses (g) for $\mathcal{R}^{J}$ ).

We turn to (IV). Assume $\mathcal{R}$ is as specified therein. We may and do assume that $\mathcal{R} \neq H$. Take a sequence of mutually orthogonal closed linear subspaces $H_{1}, H_{2}, \ldots$ of $H$ such that $\mathcal{R}=\operatorname{ran}\left[H_{1}, H_{2}, \ldots\right]$ and (23) holds. Since $\operatorname{ran}\left[H_{1}, H_{2}, \ldots\right]=\operatorname{ran}\left[\{0\}, H_{1}, H_{2}, \ldots\right]$, we may and do assume that $H_{1} \neq\{0\}$. Finally, there is a sequence of natural numbers $1=v_{1}<v_{2}<\cdots$ such that

$$
H_{v_{n}} \neq\{0\} \quad(n>0)
$$

(because $\mathcal{R} \neq H$ ). We consider each of $H_{j}=H$ in $H^{J}=\bigoplus_{j \in J} H_{j}$ as $H_{j}=\bigoplus_{n=1}^{\infty} H_{j, n}$ where $H_{j, n}=H_{n}$ for any $j \in J$ and $n>0$. Let

$$
W: H^{J}=\bigoplus_{j \in J}\left(\bigoplus_{n=1}^{\infty} H_{j, n}\right) \rightarrow \bigoplus_{n=1}^{\infty}\left(\bigoplus_{j \in J} H_{j, n}\right)
$$

be the natural unitary operator that shuffles coordinates: $W\left(\left(x_{j, n}\right)_{n=1}^{\infty}\right)_{j \in J}=$ $\left(\left(x_{j, n}\right)_{j \in J}\right)_{n=1}^{\infty}$. For simplicity, set $K_{n} \stackrel{\text { def }}{=} \bigoplus_{j \in J} H_{j, n}(n>0)$ and $K \stackrel{\text { def }}{=} \bigoplus_{n=1}^{\infty} K_{n}$. Observe that $W\left(\mathcal{R}^{J}\right)$ contains the space $\mathcal{T} \stackrel{\text { def }}{=} \operatorname{ran}\left[K_{1}, K_{2}, \ldots\right]$. So, to complete the proof of (IV), it is enough to show that $\mathcal{T}$ contains a dense (in $K$ ) operator range of type $\mathrm{R}_{\gamma}$ where $\gamma=\operatorname{card}(J) \geq \aleph_{0}$. To this end, note that $\operatorname{dim}\left(K_{v_{n}}\right)=\gamma$ for all $n>0$ (thanks to (25)). Hence, each of the spaces $K_{v_{n}}$ can be decomposed as $K_{v_{n}}=\bigoplus_{k=v_{n}}^{v_{n+1}-1} Y_{k}$ where $\operatorname{dim}\left(Y_{k}\right)=\gamma$ for any $k>0$ (recall that $v_{1}=1$ ). Now relations $K=\bigoplus_{k=1}^{\infty} Y_{k}$ and 
$\operatorname{ran}\left[Y_{1}, Y_{2}, \ldots\right] \subset \mathcal{T}$ finish the proof of (IV).

As (V) is an immediate consequence of (I)-(IV) and of the fact that all dense operator ranges of the same type are equivalent, it remains to show (VI). To this end, we start from (22) (with $\operatorname{dim}\left(H_{n}\right)=\alpha_{n}$ ) and (23). It follows from condition (b) that there is an infinite set $\Lambda \subset\{1,2, \ldots\}$ such that for any its infinite subset $\Lambda_{0}$ one has $\sum_{n \in \Lambda_{0}} \alpha_{n}=\alpha$. We divide $\Lambda$ into pairwise disjoint infinite sets $\Lambda_{1}, \Lambda_{2}, \ldots$ such that

$$
\min \left(\Lambda_{n}\right) \geq n \quad(n>0) .
$$

(We assume that $\Lambda=\bigcup_{n=1}^{\infty} \Lambda_{n}$.) Finally, for each positive $n$ define $H_{n}^{\prime}$ as follows:

- $H_{n}^{\prime}=\bigoplus_{k \in \Lambda_{n}} H_{k}$ if $n \in \Lambda$;

- $H_{n}^{\prime}=\left(\bigoplus_{k \in \Lambda_{n}} H_{k}\right) \oplus H_{n}$ otherwise.

Note that $H=\bigoplus_{n=1}^{\infty} H_{n}^{\prime}$ (by (23)) and $\operatorname{dim}\left(H_{n}^{\prime}\right)=\alpha$ for all $n$. What is more, (26) implies that $\operatorname{ran}\left[H_{1}, H_{2}, \ldots\right] \subset \operatorname{ran}\left[H_{1}^{\prime}, H_{2}^{\prime}, \ldots\right]$ which shows that $\mathcal{R}$ is contained in an operator range of type $\mathrm{R}_{\alpha}$. The final claim of (VI) is now a consequence of (V) and (II).

In a similar manner one shows the following result whose proof is left to the reader.

Proposition 6 Let $\mathcal{R}$ be a dense operator range of type $\mathrm{R}\left(\alpha_{n}\right)_{n=1}^{\infty}$ in a Hilbert space $H$ and let $\beta$ be an arbitrary cardinal number. The following conditions are equivalent:

(a) there exists a closed linear subspace $Z$ of $H$ such that $Z \cap \mathcal{R}=\{0\}$ and $\operatorname{dim}(Z)=\beta$;

(b) for any cardinal $\gamma<\beta$ there is a closed linear subspace $W$ of $H$ such that $W \cap \mathcal{R}=\{0\}$ and $\operatorname{dim}(W)>\gamma$;

(c) $\sum_{k=n}^{\infty} \alpha_{k} \geq \beta$ for any $n>0$.

(d) $\operatorname{dim}\left(K^{\perp}\right) \geq \beta$ for any linear subspace $K$ of $\mathcal{R}$ that is closed in $H$.

Recall that the structure (that is, unitary equivalence type) of the isometry $W_{Z}$ for any $Z \in \mathscr{Z}(A)$ where $A \in \mathfrak{B}_{+}(H)$ is completely determined by the cardinals $\alpha(Z)=$ $\operatorname{dim}(Z)$ and $\beta_{A}(Z)=\operatorname{dim}\left([Z]_{A}^{\perp}\right)$ (see Theorem 3 ). So, the set

$$
\Xi(A) \stackrel{\text { def }}{=}\left\{\left(\operatorname{dim}(Z), \operatorname{dim}\left([Z]_{A}^{\perp}\right)\right): Z \in \mathscr{Z}(A)\right\}
$$

contains full information on possible unitary equivalence types of members of $\mathscr{V}(A)$. Our nearest aim is to determine $\Xi(A)$. To this end, let us introduce three additional characteristic quantities:

- $\operatorname{cdr}(A) \stackrel{\text { def }}{=} \min \left\{\operatorname{dim}\left(K^{\perp}\right): K \subset \mathcal{R}(A)\right.$ is a closed linear subspace of $\left.H\right\}$;

- $\operatorname{eig}(A) \stackrel{\text { def }}{=} \operatorname{dim}(\operatorname{Eig}(A))+1$ if $\operatorname{Eig}(A)$ is finite-dimensional and $\operatorname{eig}(A)=\infty$ otherwise where $\operatorname{Eig}(A) \stackrel{\text { def }}{=} \bigoplus_{\alpha \in \mathbb{C}} \mathcal{N}(A-\alpha I)$; 
- $\operatorname{ess}(A)$ is a natural number $n$ if $A$ acts on a separable Hilbert space and $n$ is the essential supremum of the multiplicity function of $A$, otherwise (that is, if for no $n$ the previous condition is fulfilled) $\operatorname{ess}(A)=\aleph_{0}$.

It is worth noting here that $\operatorname{cdr}(A)=\min _{n>0} \sum_{k=n}^{\infty} \alpha_{k}$ if $\mathcal{R}(A)$ is of type $\mathrm{R}\left(\alpha_{n}\right)_{n=1}^{\infty}$, which simply follows from Proposition 6.

Everywhere below, $\alpha$ and $\beta$ are cardinal numbers and " $\beta<\infty$ " means that $\beta$ is finite.

Theorem 6 Let $A \in \mathfrak{B}_{+}(H)$ be non-invertible.

(I) If $H$ is separable, then $\Xi(A)$ consists of all pairs $(\alpha, \beta)$ such that

- $\alpha \leq \aleph_{0}$ and $\beta=\aleph_{0}$; or

- $\operatorname{ess}(A) \leq \alpha \leq \aleph_{0}$ and $\beta<\operatorname{eig}(A)$.

(II) If $H$ is non-separable, then $\Xi(A)$ consists of all pairs $(\alpha, \beta)$ such that

- $\alpha<\operatorname{dim}(H)$ and $\alpha \leq \operatorname{cdr}(A)$ and $\beta=\operatorname{dim}(H)$; or

- $\alpha=\operatorname{dim}(H)=\operatorname{cdr}(A)$ and $\aleph_{0} \leq \beta \leq \operatorname{dim}(H)$; or

- $\alpha=\operatorname{dim}(H)=\operatorname{cdr}(A)$ and $\beta<\operatorname{eig}(A)$.

Proof First assume $H$ is separable. The proof of Proposition 3 shows that $A$ is a direct sum of two non-invertible positive operators, say $A=B \oplus C$. Since $B$ is noninvertible, it follows that there is a closed infinite-dimensional linear subspace $Z$ of $\bar{R}(B)$ such that $Z \cap \mathcal{R}(B)=\{0\}$. Then all closed subspaces $W$ of $Z$ belong to $\mathscr{Z}(A)$ and satisfy $\operatorname{dim}\left([W]_{A}^{\perp}\right)=\aleph_{0}$ (since $C$ is non-invertible and one-to-one, its domain is infinite-dimensional). So, to finish the proof in the separable case, it is sufficient to characterize all pairs $(\alpha, \beta) \in \Xi(A)$ with finite $\beta$. Observe that if $Z \in \mathscr{Z}(A)$ is such that $\operatorname{dim}(Z)=\alpha$ and $\operatorname{dim}\left([Z]_{A}^{\perp}\right)=\beta<\aleph_{0}$, then $[Z]_{A}^{\perp} \subset \operatorname{Eig}(A)$ (recall that $\left.\operatorname{Eig}(A)=\bigoplus_{\alpha \in \mathbb{C}} \mathcal{N}(A-\alpha I)\right)$, because $[Z]_{A}^{\perp}$ is a finite-dimensional invariant subspace for $A$. Consequently, $\beta<\operatorname{eig}(A)$. Moreover, ess $\left(A\left\lceil[Z]_{A}\right) \leq \operatorname{ess}(A)\right.$ and Proposition 2 implies that $\operatorname{ess}\left(A\left\lceil[Z]_{A}\right) \leq \alpha\right.$. Conversely, if $\operatorname{ess}(A) \leq \alpha \leq \aleph_{0}$ and $\beta<\operatorname{eig}(A)$, then there is a linear subspace $E$ of $\operatorname{Eig}(A)$ of (finite) dimension $\beta$ that is invariant for $A$. Denoting by $K$ the orthogonal complement of $E$, we see that $K$ is invariant for $A$ and $\operatorname{ess}(A\lceil K) \leq \operatorname{ess}(A)$. So, ess $(A\lceil K) \leq \alpha$ and therefore-thanks to Propositions 2 and 3 -there is $Z \in \mathscr{Z}\left(A\lceil K)\right.$ such that $\operatorname{dim}(Z)=\alpha$ and $[Z]_{A}=K$. Then $\operatorname{dim}\left([Z]_{A}^{\perp}\right)=\operatorname{dim}(E)=\beta$ and hence $(\alpha, \beta) \in \Xi(A)$.

Now we turn to the non-separable case. First assume $(\alpha, \beta) \in \Xi(A)$ and choose $Z \in \mathscr{Z}(A)$ for which $\operatorname{dim}(Z)=\alpha$ and $\beta=\operatorname{dim}\left([Z]_{A}^{\perp}\right)$. It follows from the definition of $\operatorname{cdr}(A) \quad$ that $\operatorname{dim}(Z) \leq \operatorname{cdr}(A)$. If $\quad \alpha<\operatorname{dim}(H), \quad$ then $\operatorname{dim}\left([Z]_{A}\right) \leq \max \left(\alpha, \aleph_{0}\right)<\operatorname{dim}(H)$ and hence $\beta=\operatorname{dim}(H)$. In the remaining case $\alpha=\operatorname{dim}(H)=\operatorname{cdr}(A)$ and $\beta$ is either infinite or $[Z]_{A}^{\perp} \subset \operatorname{Eig}(A)$ and thus finite $\beta$ must satisfy $\beta<\operatorname{eig}(A)$. This shows that the condition specified in (II) is necessary. 
Now we show its sufficiency. To this end, we fix a pair $\alpha, \beta$ of cardinals such that $\alpha \leq \operatorname{cdr}(A)$. It follows from Proposition 6 that there is $Z \in \mathscr{Z}(A)$ with $\operatorname{dim}(Z)=\alpha$.

First assume $\alpha<\operatorname{dim}(H)$ (then $\beta=\operatorname{dim}(H)$. Choose any $Z \in \mathscr{Z}(A)$ such that $\operatorname{dim}(Z)=\alpha$. Then $\quad \operatorname{dim}\left([Z]_{A}\right) \leq \max \left(\alpha, \aleph_{0}\right)<\operatorname{dim}(H) \quad$ and thus $\operatorname{dim}\left([Z]_{A}^{\perp}\right)=\operatorname{dim}(H)=\beta$, which implies $(\alpha, \beta) \in \Xi(A)$.

Now assume $\alpha=\operatorname{dim}(H)=\operatorname{cdr}(A)$. The proof of Proposition 4 shows that $A$ can be decomposed as $A=\bigoplus_{s \in S} A_{s}$ where $\operatorname{card}(S)=\alpha$ and each of $A_{s}$ is non-invertible and acts on a separable Hilbert space. Moreover, we have shown there that the existence of such a decomposition is sufficient for the existence of $Z \in \mathscr{Z}(A)$ such that $\operatorname{dim}(Z)=\alpha$ and $[Z]_{A}=H$. We will use this property below.

If $\beta$ is infinite, we take disjoint subsets $S_{1}$ and $S_{2}$ of $S$ such that $S=S_{1} \cup S_{2}$, $\operatorname{card}\left(S_{1}\right)=\alpha$ and $\operatorname{card}\left(S_{2}\right)=\beta$. We infer from the property evoked above that there is $W \in \mathscr{Z}\left(\bigoplus_{s \in S_{1}} A_{s}\right)$ such that $\operatorname{dim}(W)=\alpha$ and $[W]_{A}$ coincides with the domain of $\bigoplus_{s \in S_{1}} A_{s}$. Then $\operatorname{dim}\left([W]_{A}^{\perp}\right)=\operatorname{card}\left(S_{2}\right)$, because $S_{2}$ is infinite and all $A_{s}$ act on separable spaces. Consequently, $(\alpha, \beta) \in \Xi(A)$.

Finally, if $\beta<\operatorname{eig}(A)$ (and still $\alpha=\operatorname{dim}(H)=\operatorname{cdr}(A)$, we can find a linear subspace $E$ of $H$ of (finite) dimension $\beta$ that is invariant for $A$. It easily follows e.g. from condition (c) of Theorem 5 that there is $Z \in \mathscr{Z}(A)$ such that $\operatorname{dim}(Z)=\alpha$ and $[Z]_{A}=E^{\perp}$. This yields $(\alpha, \beta) \in \Xi(A)$ and we are done.

\section{Unbounded positive operators}

Recall that a (densely defined) operator $T: \mathcal{D}(T) \rightarrow H$ (where $\mathcal{D}(T) \subset H$ ) is said to be positive if $\langle T x, x\rangle_{H} \geq 0$ for any $x \in \mathcal{D}(T)$. We emphasize that, according to the above definition, positive operators need not be self-adjoint.

The main aim of this section is the following consequence of the results presented in previous sections.

Theorem 7 For a (possibly unbounded) positive self-adjoint operator $A$ in a Hilbert space $H$ the following conditions are equivalent:

(i) A is a unique positive closed densely defined operator $T$ in $H$ such that $|T|=A$;

(ii) $A$ is bounded.

Proof Assume $A$ is not bounded and let $E$ denote the spectral measure of $A$. We will show that there is an isometry $V$ on $H$ such that $\operatorname{dim}\left(\mathcal{R}(V)^{\perp}\right)=1$ and $T \stackrel{\text { def }}{=} V A$ is a positive closed operator such that $|T|=A \neq T$. To this end, set $H_{0}=\mathcal{R}(E([1, \infty)))$ and $H_{1}=H_{0}^{\perp}$. A decomposes as $A=A_{0} \oplus A_{1}$ where $A_{j}$ is a positive self-adjoint operator in $H_{j}$ (for $j=1,2$ ). Moreover, $A_{0}$ is not bounded, $\left\langle A_{0} x, x\right\rangle_{H} \geq\|x\|^{2}$ for $x \in \mathcal{D}\left(A_{0}\right)$ and $\mathcal{R}\left(A_{0}\right)=H_{0}$. It is sufficient to show our claim (stated at the beginning of the proof) for $A_{0}$ (because if $V_{0}$ is an appropriate isometry for $A_{0}$, then $V_{0} \oplus I_{H_{1}}$ is appropriate for $A$ ) and thus we may and do assume that $A=A_{0}$ (and $\left.H=H_{0}\right)$. Let $B=A^{-1} \in \mathfrak{B}_{+}(H)$. It follows that $\mathcal{R}(B) \neq H$ and we infer from 
Proposition 1 that there is an isometry $V \in \mathscr{V}(B)$ such that $\operatorname{dim}\left(\mathcal{R}(V)^{\perp}\right)=1$. For any $x \in \mathcal{D}(A)$ set $y=A x$ and observe that

$$
\langle V A x, x\rangle_{H}=\langle V y, B y\rangle_{H}=\langle B V y, y\rangle_{H} \geq 0,
$$

which shows that $T=V A$ is positive. Moreover, since $A$ is closed and $V$ is isometric, $T$ is closed as well and $T^{*}=A V^{*}$. Thus $T^{*} T=A^{2}$ and consequently $|T|=A$. Finally, since $V \neq I$ and $\mathcal{R}(A)$ is dense in $H$, we have $T \neq A$. This shows that (i) is followed by (ii). The reverse implication is well-known and left to the reader as a simple exercise.

As a consequence of the above theorem, we get the following, a little bit surprising, result.

Corollary 3 For a function $u: X \rightarrow(0, \infty)$ defined on a non-empty set $X$ the following conditions are equivalent:

(i) $u$ is unbounded;

(ii) there exists a positive self-adjoint operator $A$ in $\ell_{2}(X)$ such that all the following conditions are fulfilled:

(O1) the domain of $A$ contains the canonical basis $\left\{e_{x}: x \in X\right\}$;

(O2) $\quad\left\{\frac{A e_{x}}{u(x)}: x \in X\right\}$ is an orthonormal system in $\ell_{2}(X)$;

(O3) $A\left(e_{z}\right) \neq u(z) e_{z}$ for some $z \in X$.

Proof First assume that $u$ is bounded and $A$ is a positive self-adjoint operator such that (O1) and (O2) hold. We shall show that $A e_{x}=u(x) e_{x}$ for any $x \in X$ (that is: that $A$ is bounded and diagonal in the canonical basis). For simplicity, set $f_{x} \frac{d e f}{=} \frac{A e_{x}}{u(x)}$ for $x \in X$. From $(\mathrm{O} 2)$ and the boundedness of $u$ it easily follows that $A\left\lceil\operatorname{lin}\left\{e_{x}: x \in X\right\}\right.$ is bounded. Consequently, $A$ is bounded as well. Moreover, for any $x, y \in X$ we have

$$
\begin{aligned}
\left\langle A^{2} e_{x}, e_{y}\right\rangle_{\ell_{2}(X)} & =\left\langle A e_{x}, A e_{y}\right\rangle_{\ell_{2}(X)}=u(x) \overline{u(y)}\left\langle f_{x}, f_{y}\right\rangle_{\ell_{2}(X)} \\
& =u(x) \overline{u(y)}\left\langle e_{x}, e_{y}\right\rangle_{\ell_{2}(X)}
\end{aligned}
$$

and hence $A^{2} e_{x}=u(x)^{2} e_{x}$. Since $A$ is a unique positive square root of $A^{2}$, we obtain that $A e_{x}=u(x) e_{x}$ for any $x \in X$-as claimed above.

Finally, assume $u$ is unbounded and let $B$ be the diagonal operator (with respect to the canonical basis) induced by $u$; that is, $\mathcal{D}(B)=\left\{f \in \ell_{2}(X): u f \in \ell_{2}(X)\right\}$ and $B f=u f$ for $f \in \mathcal{D}(B)$. Since $B$ is not bounded, we conclude from the proof of Theorem 7 that there is an isometry $V$ such that $\operatorname{dim}\left(\mathcal{R}(V)^{\perp}\right)=1$ and $T \stackrel{\text { def }}{=} V B$ is a positive closed operator. Hence the vectors $\frac{T\left(e_{x}\right)}{u(x)}=V\left(e_{x}\right)$ form an orthonormal system different from the canonical one. (Note also that it is possible to enlarge this system by adding a single vector to obtain an orthonormal basis of $\ell_{2}(X)$ - since $\operatorname{dim}\left(\mathcal{R}(V)^{\perp}\right)=1$.) Now to end the proof, it suffices to apply the Friedrichs' theorem 
[12] on extending positive operators (consult also, e.g., [1] or Theorem 5.38 in [24]) to get a positive self-adjoint operator $A$ in $\ell_{2}(X)$ that extends $T$ and satisfies conditions $(\mathrm{O} 1)-(\mathrm{O} 3)$.

We leave it to the reader as an exercise that whenever conditions $(\mathrm{O} 1)-(\mathrm{O} 3)$ are fulfilled (for a positive operator $A$ ), the system in $(\mathrm{O} 2)$ is never an orthonormal basis. However, as the above proof shows, if only $u$ is an unbounded function, we can always find such an operator $A$ for which the closed linear subspace generated by the system from $(\mathrm{O} 2)$ has codimension 1 .

We end this section with the following result which is unrelated with the main subject of the paper (that is, it says nothing about positivity). We will use it in the next section. It is likely that this result is already known. However, we could not find it in the literature. It can be considered interesting in its own right.

Lemma 2 Let $T$ be a closable operator and $S \stackrel{\text { def }}{=}\{x \in \mathcal{D}(T):\|T x\| \leq 1\}$.

(a) $T\lceil S$ is continuous in the weak topologies, and $S$ is closed in $\mathcal{D}(T)$.

(b) If $T$ is closed, then $S$ is a closed set.

Remark 2 Lemma 2 (and its proof without any change) is valid for all (not necessarily densely defined) closable linear operators $T: \mathcal{D}(T) \rightarrow Y$ where $\mathcal{D}(T)$ is a subspace of a Banach space $X$ and $Y$ is a reflexive Banach space.

Proof (Proof of Lemma 2) Replacing $T$ by $\bar{T}$, we may and do assume $T$ is closed. In that case we need to show that $T\lceil S$ is continuous in the weak topologies, and $S$ is closed. To this end, let $\boldsymbol{x}=\left(x_{\sigma}\right)_{\sigma \in \Sigma}$ be a net in $S$ that weakly converges to some $z \in H$ (where $H$ is the underlying space containing the domain of $T$ ). Let $\left(y_{\lambda}\right)_{\lambda \in \Lambda}$ be any subnet of $\boldsymbol{x}$ such that $\left(T y_{\lambda}\right)_{\lambda \in \Lambda}$ is weakly convergent, say to $w$. Then $\left(y_{\lambda}, T y_{\lambda}\right)_{\lambda \in \Lambda} \subset \Gamma(T)$ is a net weakly convergent (in the product space) to $(z, w)$. Since norm closed convex subsets of Banach spaces are weakly closed, we conclude that $(z, w) \in \Gamma(T)$; that is, $z \in \mathcal{D}(T)$ and $T z=w$. This shows that each weakly convergent subnet of $\left(T x_{\sigma}\right)_{\sigma \in \Sigma}$ converges to $T z$. So, it follows from the weak compactness of the unit ball (in the target space) that the net $\left(T x_{\sigma}\right)_{\sigma \in \Sigma}$ itself converges to $T z$. In particular, $\|T z\| \leq 1$ and we are done.

\section{Positive definite kernels}

Before passing to the main issue of this part, first we recall necessary notions.

A kernel on $X$ (where $X$ is an arbitrary non-empty set) is a complex-valued function on $X \times X$. A kernel $K: X \times X \rightarrow \mathbb{C}$ is said to be a positive definite kernel (on $X$ ) (or a Hilbert space reproducing kernel, or briefly a reproducing kernel) if

$$
\sum_{j, k=1}^{n} \lambda_{j} \bar{\lambda}_{k} K\left(x_{j}, x_{k}\right) \geq 0
$$

for any $n \geq 1, x_{1}, \ldots, x_{n} \in X$ and $\lambda_{1}, \ldots, \lambda_{n} \in \mathbb{C}$. Note that the above condition says that the sum on the left-hand side of (27) (whose summands are complex!) is a non- 
negative real number. It is well-known (and easy to check) that for any reproducing kernel $K$ on $X$,

$$
K(y, x)=\overline{K(x, y)}
$$

and $K(x, x) \geq 0$ for all $x, y \in X$. It is well-known (and can briefly be proved by applying Sylvester's theorem on strictly positive definite matrices) that a kernel $K: X \times X \rightarrow \mathbb{C}$ is positive definite iff $K$ satisfies (28) and $\operatorname{det}\left[K\left(x_{j}, x_{k}\right)\right]_{j, k=1}^{n} \geq 0$ for all $x_{1}, \ldots, x_{n} \in X$ (and arbitrary $n \geq 1$ ). Another equivalent (and well-known) condition for the kernel $K$ to be a reproducing kernel is the existence of a function $j: X \rightarrow H$ where $H$ is some Hilbert space such that $K(x, y)=\langle j(x), j(y)\rangle_{H}$. To shorten statements, below we will use the abbreviation "pd" for "positive definite." We will also write " $K \gg 0$ " to express that $K$ is a pd kernel. More generally, for two kernels $K$ and $L$ defined on a common set the notations " $K \ll L$ " and " $L \gg K$ " will mean that $L-K$ is a pd kernel.

For more information on reproducing kernels consult [5, 22] or [16].

In this paper we study those pd kernels that have a pdms root (see Definition 1). Note that, thanks to (28), (1) is equivalent to

$$
L(x, z)=\sum_{y \in X} K(x, y) \overline{K(z, y)} .
$$

In particular, if $z=x$, the series in (1) has non-negative summands and thus its sum is well-defined (it is either a real number or $\infty$ ). And, if all these series (with $z=x$ ) are finite, the Schwarz inequality shows that the right-hand side series in (1) converges for arbitrary $x, z \in X$. So, there is no ambiguity in understanding this formula. To simplify further statements, let us introduce the following

Definition 3 A kernel $K$ on $X$ is called an $\ell_{2}$-kernel if both $K(x, \cdot)$ and $K(\cdot, x)$ are members of $\ell_{2}(X)$ for any $x \in X$.

For two $\ell_{2}$-kernels $K$ and $L$ on $X, K * L$ is a kernel on $X$ given by

$$
(K * L)(x, z)=\sum_{y \in Y} K(x, y) L(y, z) \quad(x, z \in X) .
$$

In addition, $K^{*}$ is a kernel on $X$ such that $K^{*}(x, y)=\overline{K(y, x)}$ for any $x, y \in X$.

The paragraph preceding the above definition explains that for any $\ell_{2}$-kernels $K$ and $L$ (both on $X$ ), $K * L$ is a well-defined kernel on $X$. Moreover, we readily have $(K * L)(x, y)=\left\langle K(x, \cdot), L^{*}(y, \cdot)\right\rangle_{\ell_{2}(X)}$. It is worth noting here that, in general, $K * L$ is not an $\ell_{2}$-kernel (see, e.g. item (A) in Example 3 below). Observe also that a pd kernel $K$ on $X$ is an $\ell_{2}$-kernel iff $K(x, \cdot) \in \ell_{2}(X)$ for all $x \in X$.

Using the notation introduced in Definition 3, we can reformulate the equation defining a pdms root as follows: $K \gg 0$ is a pdms root of $L \gg 0$ iff $K$ is an $\ell_{2}$-kernel and $L=K * K$. In the last statement the assumption that $L \gg 0$ can be skipped as shown by the following very easy

Proposition 7 For any $\ell_{2}$-kernel $K, K * K^{*}$ is a pd kernel. In particular, if $K \gg 0$ is an $\ell_{2}$-kernel, then $K * K \gg 0$ as well. 
Proof Each pd kernel $K$ satisfies $K=K^{*}$, so it is sufficient to prove the first claim which follows from the formula $\left(K * K^{*}\right)(x, y)=\langle K(x, \cdot), K(y, \cdot)\rangle_{\ell_{2}(X)}$.

For any kernel $K$ on $X$ let $K_{\text {op }}$ denote a unique linear operator defined on $\ell_{\text {fin }}(X)$ such that $K_{\mathrm{op}}\left(e_{x}\right)=K(x, \cdot)$ for all $x \in X$ (so, all values of $K_{\mathrm{op}}$ are complex-valued functions on $X)$. Everywhere below its domain $\mathcal{D}\left(K_{\mathrm{op}}\right)=\ell_{\text {fin }}(X)$ will always be equipped with the norm and the topology inherited from $\ell_{2}(X)$ and considered as a subspace of $\ell_{2}(X)$. In contrast, the target space of $K_{\mathrm{op}}$ will vary and will always be specified.

Lemma 3 For any $\ell_{2}$-kernel $K$ on $X, K_{\mathrm{op}}: \ell_{\text {fin }}(X) \rightarrow \ell_{2}(X)$ is a closable operator such that $K(x, y)=\left\langle K_{\mathrm{op}} e_{x}, e_{y}\right\rangle_{\ell_{2}(X)}$ for any $x, y \in X$.

Proof The only thing that needs proving is the closability of $K_{\mathrm{op}}$. But this easily follows from the relation: $\left\langle K_{\mathrm{op}} f, e_{x}\right\rangle_{\ell_{2}(X)}=\left\langle f, K^{*}(x, \cdot)\right\rangle_{\ell_{2}(X)}\left(x \in X, f \in \ell_{\text {fin }}(X)\right)$. The details are left to the reader.

Every pd kernel $K$ on $X$ generates a unique Hilbert function space (consisting of complex-valued functions on $X$ ), to be denoted by $\mathscr{H}_{K}$, such that the following two conditions are fulfilled:

- the functions $K(x, \cdot)$ (where $x$ runs over all elements of $X$ ) belong to $\mathscr{H}_{K}$;

- $\langle f, K(x, \cdot)\rangle_{\mathscr{H}_{K}}=f(x)$ for any $x \in X$ and $f \in \mathscr{H}_{K}$.

In particular, $K(x, y)=\langle K(x, \cdot), K(y, \cdot)\rangle_{\mathscr{H}_{K}}$. (Very often $\mathscr{H}_{K}$ is defined by conditions obtained from the above two by replacing the functions $K(x, \cdot)$ by $K(\cdot, x)$. In general, this way leads to a different vector space. However, both the approaches are fully equivalent and it is a matter of taste which one to choose. Our choice is more convenient for our purposes.) A well-known fact says that $\mathscr{H}_{\delta_{X}}=\ell_{2}(X)$, which we will use many times without any additional explanations. (Recall that $\delta_{X}$ is the pd kernel on $X$ such that $\delta_{X}(x, x)=1$ for all $x \in X$ and $\delta_{X}(x, y)=0$ whenever $x \neq y$.)

To simplify further statements, let us say that an operator $T: \mathcal{D}(T) \rightarrow H$ (where $\ell_{\text {fin }}(X) \subset \mathcal{D}(T) \subset \ell_{2}(X)$ and $H$ is a Hilbert space) factorizes a pd kernel $K$ on $X$ if

$$
K(x, y)=\left\langle T e_{x}, T e_{y}\right\rangle_{H} \quad(x, y \in H) .
$$

Lemma 4 For a pd kernel $K$ on $X$ the following conditions are equivalent:

(i) there exists a closable operator that factorizes $K$;

(ii) the restriction to $\ell_{\text {fin }}(X)$ of each operator that factorizes $K$ is closable.

Proof It is easy to see that (i) is implied by (ii) (note that it is sufficient to show the existence of an operator that factorizes $K$ ): the operator $K_{\text {op }}: \ell_{\text {fin }}(X) \rightarrow \mathscr{H}_{K}$ factorizes $K$. The reverse implication is also simple: if $S: \mathcal{D}(S) \rightarrow H$ and $T$ : $\mathcal{D}(T) \rightarrow E$ factorize $K$, then $\left\langle T e_{x}, T e_{y}\right\rangle_{E}=\left\langle S e_{x}, S e_{y}\right\rangle_{H}$ for any $x, y \in X$ and therefore there exists a linear isometry $V: T\left(\ell_{\text {fin }}(X)\right) \rightarrow H$ such that $S f=V T f$ for 
any $f \in \ell_{\text {fin }}(X)$. Consequently, $S\left\lceil\ell_{\text {fin }}(X)\right.$ is closable iff $T\left\lceil\ell_{\text {fin }}(X)\right.$ is so, and we are done.

For a collection of kernels $\left\{K_{s}: X_{s} \times X_{s} \rightarrow \mathbb{C}\right\}_{s \in S}$ where the sets $X_{s}$ are all pairwise disjoint we define the kernel $\bigoplus_{s \in S} K_{s}$ on the (disjoint) union $\bigsqcup_{s \in S} X_{s}$ of $X_{s}$ as follows:

- $\left(\bigoplus_{s \in S} K_{s}\right)(x, y)=K_{t}(x, y)$ for $x, y \in X_{t}$ and arbitrary $t \in S$

- $\left(\oplus_{s \in S} K_{s}\right)(x, y)=0$ if $x \in X_{p}, y \in X_{q}$ and $p \neq q$.

It is easy to check and left to the reader that $\bigoplus_{s \in S} K_{s} \gg 0$ iff $K_{s} \gg 0$ for all $s \in S$.

For simplicity, let us call a vector $u \in \ell_{\text {fin }}(X) K$-unit (where $K$ is a pd kernel on $X)$ if $\sum_{x, y \in X} u(x) \overline{u(y)} K(x, y) \leq 1$.

Now we gather various criteria for a pd kernel to have a pdms root.

Theorem 8 For a pd kernel $K$ on $X$ the following conditions are equivalent:

(a1) $K$ has a pdms root;

(a2) there exists an $\ell_{2}$-kernel $L$ on $X$ such that $K=L * L^{*}$;

$\mathrm{b} \quad$ the set $X$ admits a decomposition $X=\bigsqcup_{s \in S} X_{s}$ into (pairwise disjoint) nonempty (at most) countable sets such that $K=\bigoplus_{s \in S} K\left\lceil X_{s} \times X_{s}\right.$ and each of $K\left\lceil X_{s} \times X_{s}\right.$ has a pdms root;

(c1) there exists a closable operator that factorizes $K$;

(c2) there exists a positive self-adjoint operator $B$ in $\ell_{2}(X)$ that factorizes $K$ and has $\ell_{\text {fin }}(X)$ as a core;

(c3) $K_{\mathrm{op}}: \ell_{\text {fin }}(X) \rightarrow \mathscr{H}_{K}$ is closable;

$\mathrm{d} \quad \ell_{2}(X) \cap \mathscr{H}_{K}$ is dense in $\mathscr{H}_{K}$;

(e1) whenever a sequence $\left(\alpha_{n}\right)_{n=1}^{\infty} \subset \ell_{\text {fin }}(X)$ norm converges to 0 and consists of $K$-unit vectors, then $\lim _{n \rightarrow \infty} \sum_{x \in X} \alpha_{n}(x) K(x, z)=0$ for all $z \in X$;

(e2) for any $z \in X$ and $\varepsilon>0$ there is a finite non-empty set $F \subset X$ such that the following condition holds: if a $K$-unit vector $f \in \ell_{\text {fin }}(X)$ vanishes at each point of $F$ and has norm not exceeding 1, then $\left|\sum_{x \in X} f(x) K(x, z)\right| \leq \varepsilon$;

(e3) for any $z \in X$ and $\varepsilon>0$ there are a finite orthonormal system $u_{1}, \ldots, u_{k}$ in $\ell_{2}(X)$ and $\delta>0$ such that the following condition is fulfilled: if a K-unit vector $v \in \ell_{\text {fin }}(X)$ satisfies $\left|\left\langle v, u_{j}\right\rangle_{\ell_{2}(X)}\right| \leq \delta$ for $j=1, \ldots, k$, then $\left|\sum_{x \in X} v(x) K(x, z)\right| \leq \varepsilon$.

Proof For the reader's convenience, let us draw a scheme of the proof: (a1) $\Rightarrow(\mathrm{c} 1) \Rightarrow(\mathrm{c} 2) \Rightarrow(\mathrm{a} 1) ; \quad(\mathrm{a} 1) \Rightarrow(\mathrm{a} 2) \Rightarrow(\mathrm{c} 1)$ $(\mathrm{c} 1) \Longleftrightarrow(\mathrm{c} 3) \Longleftrightarrow(\mathrm{d})$; $(\mathrm{c} 3) \Rightarrow(\mathrm{e} 3) \Rightarrow(\mathrm{e} 2) \Rightarrow(\mathrm{e} 1) \Rightarrow(\mathrm{c} 3)$ and finally $(\mathrm{a} 1) \Rightarrow(\mathrm{b}) \Rightarrow(\mathrm{a} 1)$.

First assume $L \gg 0$ satisfies $K=L * L$ (see (a1)). This means that

$$
L_{\mathrm{op}}: \ell_{\text {fin }}(X) \rightarrow \ell_{2}(X)
$$

factorizes $K$. It follows from Lemma 3 that $L_{\mathrm{op}}$ is closable (recall that $L$ is an $\ell_{2}$ kernel). So, (c1) holds. Let us now check that (c2) is implied by (c1). To this end, let 
$T: \ell_{f i n}(X) \rightarrow H$ be closable and factorize $K$, and let $\bar{T}=Q B$ be the polar decomposition of $\bar{T}$. Then $B$ is a positive self-adjoint operator whose domain contains $\ell_{\text {fin }}(X)$ and $Q$ is isometric on $\overline{\mathcal{R}}(B)$. So, $K(x, y)=$ $\left\langle T e_{x}, T e_{y}\right\rangle_{H}=\left\langle Q B e_{x}, Q B e_{y}\right\rangle_{H}=\left\langle B e_{x}, B e_{y}\right\rangle_{\ell_{2}(X)}$. Moreover, since $\ell_{\text {fin }}(X)$ is a core of $\bar{T}$, it is a core of $B$ as well-that is, (c2) holds. Now we will show that (a1) follows from (c2). So, let $B$ be as specified in (c2) and define $L: X \times X \rightarrow \mathbb{C}$ by $L(x, y)=\left\langle B e_{x}, e_{y}\right\rangle_{\ell_{2}(X)}(x, y \in X)$. Since $B$ is positive, it is readily seen that $L \gg 0$. Moreover, observe that $B e_{x}=\sum_{y \in X} L(x, y) e_{y}$ and therefore $L$ is an $\ell_{2}$-kernel and $\langle L(x, \cdot), L(y, \cdot)\rangle_{\ell_{2}(X)}=\left\langle B e_{x}, B e_{y}\right\rangle_{\ell_{2}(X)}=K(x, y)$ and (a1) is fulfilled.

Of course, (a1) is followed by (a2). Conversely, if $L$ is as specified in (a2), then $L_{\mathrm{op}}: \ell_{\text {fin }}(X) \rightarrow \ell_{2}(X)$ is closable (by Lemma 3 ) and factorizes $K$, which shows (c1).

Further, if (c1) holds, then $K_{\mathrm{op}}: \ell_{\text {fin }}(X) \rightarrow \mathscr{H}_{K}$ is closable as it factorizes $K$ (see Lemma 4 and its proof), which shows that (c1) implies (c3). The reverse implication is trivial. To show that (c3) is equivalent to (d), consider $T=K_{\mathrm{op}}: \ell_{\text {fin }}(X) \rightarrow \mathscr{H}_{K}$ and recall that (since $T$ is densely defined) $T$ is closable iff $T^{*}$ is densely defined. Therefore it is sufficient to check that $\mathcal{D}\left(T^{*}\right)=\ell_{2}(X) \cap \mathscr{H}_{K}$. To this end, observe that $g \in \mathscr{H}_{K}$ belongs to $\mathcal{D}\left(T^{*}\right)$ and $T^{*} g=f \in \ell_{2}(X)$ iff $\left\langle T e_{x}, g\right\rangle_{\mathscr{H}_{K}}=\overline{f(x)}$ for all $x \in X$. Equivalently, we need to have $f(x)=\langle g, K(x, \cdot)\rangle_{\mathscr{H}_{K}}=g(x)$. So, $g \in \mathcal{D}\left(T^{*}\right)$ iff $g \in \ell_{2}(X) \cap \mathscr{H}_{K}$ (and then $T^{*} g=g$ ), which finishes this part of the proof.

Going further, for simplicity, we denote by $S \subset \ell_{\text {fin }}(X)$ the set of all $K$-unit vectors, and by $T$ the operator $K_{\mathrm{op}}: \ell_{2}(X) \rightarrow \mathscr{H}_{K}$. Observe that for any $u \in \ell_{\text {fin }}(X)$,

$$
u \in S \Longleftrightarrow\|T u\| \leq 1
$$

and $\sum_{x \in X} u(x) K(x, y)=\langle T u, K(y, \cdot)\rangle_{\mathscr{H}_{K}}$ (for any $y \in X$ ). Now if (c3) holds, it follows from Lemma 2 that (e3) is fulfilled. Indeed, a note that all sets of the form

$$
\left\{v \in \ell_{\text {fin }}(X):\left|\left\langle v, u_{j}\right\rangle\right|<\delta(j=1, \ldots, k)\right\}
$$

(where $\delta>0$ and $u_{1}, \ldots, u_{k}$ is a finite orthonormal system in $\ell_{2}(X)$ ) form a neighbourhood basis of 0 in the weak topology of $\ell_{2}(X)$ enables one deducing (e3) from (c3) (we leave the details to the reader). Further, if (e3) holds, then (e2) holds as well (for in (e2) $K$-unit vectors are taken from the unit ball of $\ell_{\text {fin }}(X)$ and on the unit ball of $\ell_{2}(X)$ a neighbourhood basis of 0 in the weak topology can be formed by the sets (30) where all vectors $u_{1}, \ldots, u_{k}$ are taken from the canonical basis of $\ell_{2}(X)$ ). Let us now give a more detailed proof that (e1) follows from (e2). To this end, assume (e2) holds and let a sequence $\left(\alpha_{n}\right)_{n=1}^{\infty}$ be as specified in (e1). Fix $z \in X$ and $\varepsilon>0$. Choose a finite set $F \subset X$ guaranteed by (e2) (for these $z$ and $\varepsilon>0$ ). Denote by $E$ the linear span of all $e_{x}$ with $x \in F$ and write $\alpha_{n}=\beta_{n}+\gamma_{n}$ where $\beta_{n} \in E$ and $\gamma_{n} \perp E$. Then $\lim _{n \rightarrow \infty} \beta_{n}=\lim _{n \rightarrow \infty} \gamma_{n}=0$. Since $E$ is finitedimensional,

$$
\lim _{n \rightarrow \infty} T \beta_{n}=0
$$


Therefore $\beta_{n} \in S$ for sufficiently large $n$. Consequently, $\frac{1}{2} \gamma_{n}=\frac{1}{2}\left(\alpha_{n}-\beta_{n}\right)$ eventually belongs to $S$ as well. Since $\gamma_{n}$ vanishes at each point of $F$, we infer from (e2) that $\left|\left\langle T \gamma_{n}, K(z, \cdot)\right\rangle_{\mathscr{H}_{K}}\right| \leq 2 \varepsilon$ for sufficiently large $n$. This inequality combined with (31) yields $\left|\left\langle T \alpha_{n}, K(z, \cdot)\right\rangle_{\mathscr{H}_{K}}\right| \leq 3 \varepsilon$ (for sufficiently large $n$ ) and hence (e1) is fulfilled. Finally, assume (e1) holds. Our aim is to show that (c3) is fulfilled; that is, that $T$ (defined above) is closable. To this end, assume $\left(\alpha_{n}\right)_{n=1}^{\infty} \subset \ell_{f i n}(X)$ norm converges to 0 and $T \alpha_{n} \rightarrow \beta \in \mathscr{H}_{K}(n \rightarrow \infty)$. We need to check that $\beta=0$. Without loss of generality, we may and do assume that $\left\|T \alpha_{n}\right\| \leq 1$. So, $\alpha_{n} \in S$ and it follows from (e1) that $\lim _{n \rightarrow \infty}\left\langle T \alpha_{n}, K(z, \cdot)\right\rangle_{\mathscr{H}_{K}}=0$ for any $z \in X$, from which it easily follows that $\beta=0$.

It remains to check that (b) is equivalent to (a1). First assume (a1) holds and choose any pd $\ell_{2}$-kernel $L$ such that $K=L * L$. Define an equivalence relation " $\sim$ " on $X$ as follows: $x \sim y$ if there are points $a_{0}, \ldots, a_{N} \in X$ (for some $N>0$ ) such that $a_{0}=x, a_{N}=y$ and $L\left(x_{j-1}, x_{j}\right) \neq 0$ for $j=1, \ldots, N$. Observe that all equivalence classes $[x]_{\sim}$ are at most countable (because the set $\{x \in X: L(x, y) \neq 0\}$ is such for any $y \in X)$. So, we can divide $X$ into pairwise disjoint sets $X_{s}$ such that $L(x, y)=0$ for any $x \in X_{s}$ and $y \in X_{s^{\prime}}$ with distinct $s, s^{\prime} \in S$ (namely, $\left\{X_{s}: s \in S\right\}=\left\{[x]_{\sim}: x \in X\right\}$ ). For simplicity, set $L_{s} \stackrel{\text { def }}{=} L\left\lceil X_{s} \times X_{s}\right.$ and note that $L=\bigoplus_{s \in S} L_{s}$ and each of $L_{s}$ is a pd $\ell_{2}$-kernel. It is then easy to verify that $L * L=$ $\bigoplus_{s \in S}\left(L_{s} * L_{s}\right)$ and therefore (b) holds. Conversely, if (b) is satisfied, then for each $s \in S$ we can choose a pd $\ell_{2}$-kernel $L_{s}$ on $X_{s}$ such that $K\left\lceil X_{s} \times X_{s}=L_{s} * L_{s}\right.$. Then it suffices to set $L \stackrel{\text { def }}{=} \bigoplus_{s \in S} L_{s}$ to get a pd $\ell_{2}$-kernel such that $K=L * L$.

In Proposition 8 below we will show that the operator $B$ witnessing the above condition (c2) is unique.

In the next theorem we will make use of the following two results. The former of them is well-known (see, e.g., Theorem 6 on page 37 in [18]) and it is likely that so is the latter, but we could not find it in the literature and thus we give its proof.

Lemma 5 For two pd kernels $K$ and $L$ on a common set and a constant $c \geq 0$ the following conditions are equivalent:

(i) $\mathscr{H}_{K} \subset \mathscr{H}_{L}$ and the identity operator from $\mathscr{H}_{K}$ into $\mathscr{H}_{L}$ has norm not exceeding $c$;

(ii) $\quad K \ll c^{2} L$.

Moreover, $\mathscr{H}_{K} \subset \mathscr{H}_{L}$ iff (ii) holds for some $c>0$.

Lemma 6 Let $\left\{K_{\sigma}\right\}_{\sigma \in \Sigma}$ be an increasing net of pd kernels on $X$, that is:

- $(\Sigma, \leq)$ is a directed set;

- $K_{\sigma} \ll K_{\tau}$ for any $\sigma, \tau \in \Sigma$ with $\sigma \leq \tau$.

If $K: X \times X \rightarrow \mathbb{C}$ is the pointwise limit of this net-that is, if

$$
K(x, y)=\lim _{\sigma \in \Sigma} K_{\sigma}(x, y) \quad(x, y \in X),
$$


then $\bigcup_{\sigma \in \Sigma} \mathscr{H}_{K_{\sigma}}$ is a dense linear subspace of $\mathscr{H}_{K}$.

Proof For simplicity and to avoid confusion, for any $\sigma \in \sum$ we denote by $\langle\cdot,-\rangle_{\sigma}$ and $\|\cdot\|_{\sigma}$ the scalar product and, respectively, the norm of $\mathscr{H}_{K_{\sigma}}$; whereas $\langle\cdot,-\rangle$ and $\|\cdot\|$ stands for the scalar product and the norm of $\mathscr{H}_{K}$. Additionally, we set $H \stackrel{\text { def }}{=} \bigcup_{\sigma \in \Sigma} \mathscr{H}_{K_{\sigma}}$.

Since $K_{\sigma} \ll K_{\tau}$ whenever $\sigma \leq \tau$, after passing with $\tau$ to the limit, we easily get $K_{\sigma} \ll K$. It follows from Lemma 5 that for any $\sigma, \tau \in \Sigma$ :

- $\mathscr{H}_{K_{\sigma}} \subset \mathscr{H}_{K_{\tau}}$ whenever $\sigma \leq \tau$; consequently, since $\Sigma$ is directed, $H$ is a linear space;

- $\mathscr{H}_{K_{\sigma}} \subset \mathscr{H}_{K}$ and $\left\|I_{\sigma}\right\| \leq 1$ where $I_{\sigma}: \mathscr{H}_{K_{\sigma}} \rightarrow \mathscr{H}_{K}$ is the identity operator.

Fix $x \in X$. Then

$$
\left\|K_{\sigma}(x, \cdot)\right\|^{2} \leq\left\|K_{\sigma}(x, \cdot)\right\|_{\sigma}^{2}=\left\langle K_{\sigma}(x, \cdot), K_{\sigma}(x, \cdot)\right\rangle_{\sigma}=K_{\sigma}(x, x) \leq K(x, x) .
$$

So, the net $\left\{K_{\sigma}(x, \cdot)\right\}_{\sigma \in \Sigma} \subset H$ is bounded in $\mathscr{H}_{K}$. Moreover, for any $z \in X$ we have

$$
\lim _{\sigma \in \Sigma}\left\langle K_{\sigma}(x, \cdot), K(z, \cdot)\right\rangle=\lim _{\sigma \in \Sigma} K_{\sigma}(x, z)=K(x, z)=\langle K(x, \cdot), K(z, \cdot)\rangle .
$$

Since the functions $K(z, \cdot)(z \in X)$ form a total subset of $\mathscr{H}_{K}$, it follows from the above convergence and the boundedness of the net under consideration that $K_{\sigma}(x, \cdot)$ weakly converge to $K(x, \cdot)$. Therefore $K(x, \cdot)$ belongs to the weak closure of $H$ which coincides with the norm closure of $H$. Consequently, $\mathscr{H}_{K}=\overline{\operatorname{lin}}\{K(x, \cdot)$ : $x \in X\}$ is contained in the (norm) closure of $H$ and we are done.

As a consequence of Theorem 8, we obtain the following

Theorem 9 Let $K$ be a pd kernel on $X$.

(I) If $K$ has a pdms root and $A$ is a non-empty subset of $X$, then $K\lceil A \times A$ has a pdms root as well.

(II) If $K=\bigoplus_{s \in S} K_{s}$, then $K$ has a pdms root iff each of $K_{s}$ has a pdms root.

(III) If $K$ has a pdms root and $u: X \rightarrow \mathbb{C}$ is a bounded function, then the kernel $L: X \times X \ni(x, y) \mapsto \overline{u(x)} u(y) K(x, y) \in \mathbb{C}$ has a pdms root as well. In particular, the kernel

$$
K_{\mathrm{bd}}: X \times X \ni(x, y) \mapsto \frac{K(x, y)}{\sqrt{\max (K(x, x), 1) \cdot \max (K(y, y), 1)}} \in \mathbb{C}
$$

has a pdms root provided so has $K$.

(IV) If $K$ is bounded and has a pdms root, then for any $x \in X, K(x, \cdot)$ is a $c_{0^{-}}$ function; that is, for any $x \in X$ and $\varepsilon>0$ there is a finite set $F \subset X$ such that $|K(x, y)|<\varepsilon$ for any $y \notin F$.

(V) If $K$ is an $\ell_{2}$-kernel, it has a pdms root. In particular, each pdms root of a pd kernel has a pdms root.

(VI) If $K=\sum_{s \in S} K_{s}$ where $\left\{K_{s}\right\}_{s \in S}$ is an arbitrary family of pd kernels having pdms roots, then $K$ also has a pdms root. 
(VII) If $K$ is the pointwise limit of an increasing net of pd kernels (cf. the statement of Lemma 6) each of which has a pdms root, then also $K$ has a pdms root.

(VIII) If $L$ is a pd kernel on $X$ such that $c_{1} K \ll L \ll c_{2} K$ for some positive constants $c_{1}$ and $c_{2}$, then either both $K$ and $L$ have pdms roots or none of them.

(IX) There exists a unique pd kernel $K_{0} \ll K$ that has a pdms root and is the greatest kernel with these properties; that is, if $L \ll K$ is a pd kernel that has a pdms root, then $L \ll K_{0}$.

(X) There exists a pd kernel $K_{1} \ll K$ that has a unique pdms root and the following property: whenever $L \gg 0$ is such that $L \ll a K$ for some constant $a>0$, then $L$ has a unique pdms root iff $L \ll b K_{1}$ for some constant $b>0$.

Before giving a proof, we explain how to understand the sum appearing in item (VI) above and when this (generalized) series converges. The formula $K=\sum_{s \in S} K_{s}$ is understood pointwise: we only assume that $K(x, y)=\sum_{s \in S} K_{s}(x, y)$ for any $x, y \in X$. In particular,

$$
\sum_{s \in S} K_{s}(x, x)<\infty \quad(x \in X)
$$

(all summands in (33) are non-negative and hence the series is well-defined as a quantity in $[0, \infty])$. Conversely, if (33) is fulfilled, then $\sum_{s \in S} K_{s}(x, y)$ is absolutely convergent (for any $x, y \in X$ ):

$$
\sum_{s \in S}\left|K_{s}(x, y)\right| \leq \sum_{s \in S} \sqrt{K_{s}(x, x) K_{s}(y, y)} \leq \sum_{s \in S}\left(K_{s}(x, x)+K_{s}(y, y)\right)<\infty
$$

where the first inequality above follows from the property that the matrix

$$
\left(\begin{array}{ll}
K(x, x) & K(x, y) \\
K(y, x) & K(y, y)
\end{array}\right)
$$

is positive.

Proof (Proof of Theorem 9) Since the restriction of a closable operator is closable as well, item (I) immediately follows from condition (c1) in Theorem 8, whereas (II) is a consequence of (I) and of item (b) therein.

To prove that $L$ defined in (III) has a pdms root, we use condition (c1) of Theorem 8. Since $K$ has a pdms kernel, there is a closable operator

$$
T: \ell_{\text {fin }}(X) \rightarrow H
$$

that factorizes $K$. Let $S: \ell_{f i n}(X) \rightarrow \ell_{f i n}(X)$ be given by $S e_{x} \stackrel{\text { def }}{=} u(x) e_{x}$. Then $S$ is bounded (since so is $u$ ) and hence the operator $T S: \ell_{\text {fin }}(X) \rightarrow H$ is closable. Observe that $T S$ factorizes $L$ and thus $L$ has a pdms root (by (c1)). The claim about $K_{\text {bd }}$ follows from the boundedness of the function 
$X \ni x \mapsto \max (K(x, x), 1)^{-1 / 2} \in(0, \infty)$.

We turn to (IV). Assume $K$ is bounded and has a pdms root. It is sufficient to show that for any $x \in X, \lim _{n \rightarrow \infty} K\left(x, y_{n}\right)=0$ for any one-to-one sequence $\left(y_{n}\right)_{n=1}^{\infty} \subset X$. To this end, take an upper bound $M \geq 1$ of $|K|$ and apply condition (e2) (in Theorem 8) to $z=x$ and $f=\frac{1}{M} e_{y_{n}}$ : (e2) implies that $\lim _{n \rightarrow \infty} \frac{1}{M} K\left(y_{n}, x\right)=0$. Consequently, $K\left(x, y_{n}\right)=\overline{K\left(y_{n}, x\right)}$ converges to 0 as $n$ tends to $\infty$.

Now assume $K$ is an $\ell_{2}$-kernel. Then $\operatorname{lin}\{K(x, \cdot): x \in X\} \subset \ell_{2}(X) \cap \mathscr{H}_{K}$ and hence $\ell_{2}(X) \cap \mathscr{H}_{K}$ is dense in $\mathscr{H}_{K}$. So, condition (d) of Theorem 8 shows that $K$ has a pdms root. Since each pdms root is an $\ell_{2}$-kernel, the whole conclusion of (V) follows.

To prove (VI) we apply condition (c1) of Theorem 8. So, for any $s \in S$ there exists a closable operator $T_{s}: \ell_{\text {fin }}(X) \rightarrow H_{s}$ that factorizes $K_{s}$. Then, for any $x \in X$, $\sum_{s \in S}\left\|T_{s} e_{x}\right\|^{2}=\sum_{s \in S} K_{s}(x, x)=K(x, x)<\infty$ and therefore $\oplus_{s \in S} T_{s} e_{x} \in \bigoplus_{s \in S} H_{s}$. In particular, for any $f \in \ell_{f i n}(X), T f \stackrel{\text { def }}{=} \oplus_{s \in S} T_{s} f \in \bigoplus_{s \in S} H_{s}$. In this way we have defined a linear operator $T: \ell_{\text {fin }}(X) \rightarrow H \stackrel{\text { def }}{=} \bigoplus_{s \in S} H_{s}$. It is readily seen that $T$ is closable_as all $T_{s}$ are such. Moreover, for any $x, y \in X$ we have

$$
\left\langle T e_{x}, T e_{y}\right\rangle_{H}=\sum_{s \in S}\left\langle T_{s} e_{x}, T_{s} e_{y}\right\rangle_{H_{s}}=\sum_{s \in S} K_{s}(x, y)=K(x, y)
$$

which shows that $T$ factorizes $K$. So, an application of (c1) from Theorem 8 completes the proof of (VI).

We turn to (VII). Let $\left\{K_{\sigma}\right\}_{\sigma \in \Sigma}$ be an increasing net (of pd kernels with pdms roots) whose pointwise limit is $K$. For simplicity, we set $H_{\sigma} \stackrel{\text { def }}{=} \mathscr{H}_{K_{\sigma}}(\sigma \in \Sigma)$ and $H \stackrel{\text { def }}{=} \mathscr{H}_{K}$. It follows from Lemma 6 that $H_{*} \stackrel{\text { def }}{=} \bigcup_{\sigma \in \Sigma} H_{\sigma}$ is a dense subspace of $H$. Moreover, Lemma 5 yields that the identity operator $I_{\sigma}: H_{\sigma} \rightarrow H$ is continuous; whereas condition (d) of Theorem 8 implies that $\ell_{2}(X) \cap H_{\sigma}$ is dense in $H_{\sigma}$. Consequently, $I_{\sigma}\left(\ell_{2}(X) \cap H_{\sigma}\right)$ is dense in $I_{\sigma}\left(H_{\sigma}\right)$ and therefore the closure of $\ell_{2}(X) \cap H$ (in $H$ ) contains $H_{*}$, which finishes the proof of (VII).

Property (VIII) immediately follows from Lemma 5 (which implies that under the assumption of (VIII), $\mathscr{H}_{K}=\mathscr{H}_{L}$ and their topologies coincide) and condition (d) of Theorem 8 (since in that case $\ell_{2}(X) \cap \mathscr{H}_{K}=\ell_{2}(X) \cap \mathscr{H}_{L}$ ).

To prove (IX), denote by $P$ the orthogonal projection from $\mathscr{H}_{K}$ onto the closure $E$ (in $\mathscr{H}_{K}$ ) of $\ell_{2}(X) \cap \mathscr{H}_{K}$ and define $K_{0}$ as follows:

$$
K_{0}(x, y)=\langle P K(x, \cdot), P K(y, \cdot)\rangle_{\mathscr{H}_{K}} \quad(x, y \in X) .
$$

Then $K_{0}$ is a pd kernel such that $\mathscr{H}_{K_{0}}=E$ and the inner product of $\mathscr{H}_{K_{0}}$ coincides with the one on $E$ inherited from $\mathscr{H}_{K}$ (consult, e.g., Theorem 5 on page 37 in [18]). In particular, $\ell_{2}(X) \cap \mathscr{H}_{K_{0}}$ is dense in $\mathscr{H}_{K_{0}}$ (and hence $K_{0}$ has a pdms root-see (d) in Theorem 8) and Lemma 5 implies that $K_{0} \ll K$. Now assume $L \gg 0$ has a pdms root and satisfies $L \ll K$. Again:

- condition (d) of Theorem 8 yields that $\ell_{2}(X) \cap \mathscr{H}_{L}$ is dense in $\mathscr{H}_{L}$; 
- Lemma 5 implies that $\mathscr{H}_{L} \subset \mathscr{H}_{K}$ and the identity operator $I: \mathscr{H}_{L} \rightarrow \mathscr{H}_{K}$ has norm not exceeding 1 .

It follows from the former of the above properties that $I\left(\ell_{2}(X) \cap \mathscr{H}_{L}\right)$ is dense in $I\left(\mathscr{H}_{L}\right)$. Consequently, $\mathscr{H}_{L}=I\left(\mathscr{H}_{L}\right)$ is contained in the closure (in $\mathscr{H}_{K}$ ) of $\ell_{2}(X) \cap \mathscr{H}_{L}$. So, $\mathscr{H}_{L} \subset E=\mathscr{H}_{K_{0}}$. Since $\|I\| \leq 1$, we obtain $L \ll K_{0}$ (one more time by Lemma 5). The maximality of $K_{0}$ (just proved) implies the uniqueness of $K_{0}$.

Finally, we turn to $(\mathrm{X})$. We will use here Theorem 2 (that has not been proved yet!). A careful reader will verify that the proof of that theorem presented below is independent of this part of the present result. Equip the vector space $H \stackrel{\text { def }}{=} \ell_{2}(X) \cap$ $\mathscr{H}_{K}$ with the inner product

$$
\langle u, v\rangle_{H} \stackrel{\text { def }}{=}\langle u, v\rangle_{\ell_{2}(X)}+\langle u, v\rangle_{\mathscr{H}_{K}} \quad(u, v \in H) .
$$

It is a kind of folklore that the above $H$ is a Hilbert space on which all the evaluation functionals (that is, all functions of the form $u \mapsto u(x)$ where $x \in X$ ) are continuous (actually both these properties are easy to prove). This means that $H$ has a reproducing kernel, say $K^{\prime}$. Since then $\mathscr{H}_{K^{\prime}}=H \subset \mathscr{H}_{K}$, it follows from Lemma 5 that $K^{\prime} \ll c K$ for some constant $c>0$. We define $K_{1}$ as $\frac{1}{c} K^{\prime}$. Observe that $K_{1} \ll K$ and $\mathscr{H}_{K_{1}}=H \subset \ell_{2}(X)=\mathscr{H}_{\delta_{X}}$. Another application of Lemma 5 yields that $K_{1} \ll c^{\prime} \delta_{X}$ for some constant $c^{\prime}>0$. So, Theorem 2 implies that $K_{1}$ has a unique pdms root. Now let $L \gg 0$ be such that

$$
L \ll a K
$$

for some constant $a>0$ and $L$ has a unique pdms root. Then-again by Theorem $2-L \ll a^{\prime} \delta_{X}$ for some constant $a^{\prime}>0$. The last property combined with Lemma 5 gives $\mathscr{H}_{L} \subset \mathscr{H}_{\delta_{X}}=\ell_{2}(X)$, whereas, similarly, (34) yields $\mathscr{H}_{L} \subset \mathscr{H}_{K}$. So, $\mathscr{H}_{L} \subset H=\mathscr{H}_{K_{1}}$ and another application of Lemma 5 leads to the final conclusion: there exists constant $b>0$ such that $L \ll b K_{1}$.

The next result gives a description of all pdms roots of a pd kernel (that has such a root).

Proposition 8 Let $K$ be a pd kernel that has a pdms root.

(I) There is a unique positive self-adjoint operator $B$ in $\ell_{2}(X)$ that factorizes $K$ and has $\ell_{\text {fin }}(X)$ as a core.

(II) If $B$ is as specified in (I), then there is a one-to-one correspondence $\kappa$ : $\mathscr{V} \rightarrow \mathscr{R}$ between the set $\mathscr{V}$ of all linear isometries $V: \overline{\mathcal{R}}(B) \rightarrow \ell_{2}(X)$ such that the operator $V B$ is positive, and the set $\mathscr{R}$ of all pdms roots of $K ; \kappa$ is given by the rule:

$$
(\kappa(V))(x, y)=\left\langle V B e_{x}, e_{y}\right\rangle_{\ell_{2}(X)} \quad(x, y \in X) .
$$

Proof The existence of the operator $B$ with all properties (apart from the uniqueness) specified in (I) follows from condition (c2) in Theorem 8 . We fix it and 
after proving (II) we will show its uniqueness.

We turn to (II). Fix for a moment $V \in \mathscr{V}$. It is easy to check that $\kappa(V)$ given by (35) is a pd kernel (because $V B$ is positive). Moreover, we have $\kappa(V)(x, \cdot)=V B e_{x}$ and hence $\kappa(V)$ is an $\ell_{2}$-kernel such that $(\kappa(V) * \kappa(V))(x, y)=$ $\left\langle V B e_{x}, V B e_{y}\right\rangle_{\ell_{2}(X)}=\left\langle B e_{x}, B e_{y}\right\rangle_{\ell_{2}(X)}=K(x, y)$. So, $\kappa(V) \in \mathscr{R}$.

Since members of $\mathscr{V}$ are defined (only) on $\overline{\mathcal{R}}(B)$ and $\ell_{\text {fin }}(X)$ is a core of $B$, we readily conclude that $\kappa$ is one-to-one. So, to end the proof of (II), it remains to check the surjectivity of $\kappa$. To this end, let $L$ be a pdms root of $K$. We consider $L_{\mathrm{op}}$ with target space $\ell_{2}(X)$. Observe that for any $x, y \in X$, $\left\langle L_{\mathrm{op}} e_{x}, L_{\mathrm{op}} e_{y}\right\rangle_{\ell_{2}(X)}=K(x, y)=\left\langle B e_{x}, B e_{y}\right\rangle_{\ell_{2}(X)}$. This equation implies that there is a linear isometry $V: \overline{B\left(\ell_{\text {fin }}(X)\right)} \rightarrow \ell_{2}(X)$ such that $L_{\mathrm{op}} f=V(B f)$ for any $f \in \ell_{\text {fin }}(X)$. Since $\ell_{\text {fin }}(X)$ is a core for $B$, we get that $\mathcal{D}(V)=\overline{\mathcal{R}}(B)$ and $V B$ is positive (as it is positive on $\ell_{\text {fin }}(X)$. Consequently, $V \in \mathscr{V}$ and

$$
(\kappa(V))(x, y)=\left\langle V B e_{x}, e_{y}\right\rangle_{\ell_{2}(X)}=\left\langle L_{\mathrm{op}} e_{x}, e_{y}\right\rangle_{\ell_{2}(X)}=L(x, y) \quad(x, y \in X) .
$$

Having (II), we can briefly validate the uniqueness of $B$. Assume $A$ is a positive selfadjoint operator in $\ell_{2}(X)$ that factorizes $K$ and has $\ell_{2}(X)$ as a core. Then $\left\langle A e_{x}, A e_{y}\right\rangle_{\ell_{2}(X)}=K(x, y)=\left\langle B e_{x}, B e_{y}\right\rangle_{\ell_{2}(X)}$ for any $x, y \in X$ and it follows from the previous paragraph that $A\left\lceil\ell_{\text {fin }}(X)=V B \backslash \ell_{\text {fin }}(X)\right.$ for some $V \in \mathscr{V}$. Since $V$ is isometric and $\ell_{\text {fin }}(X)$ is a core for both $A$ and $B$, we obtain $A=V B$. Hence $\mathcal{N}(A)=\mathcal{N}(B)$. Extend $V$ to the partial isometry $Q$ such that $\mathcal{N}(Q)=\mathcal{N}(B)$. Then also $A=Q B$ and it follows from the uniqueness of the polar decomposition that $A=B$.

As an immediate consequence we obtain the following result (we skip its proof).

Corollary 4 For any pd kernel that has a pdms root there exists a unique pdms root $K$ such that the closure of $K_{\mathrm{op}}: \ell_{\text {fin }}(X) \rightarrow \ell_{2}(X)$ is self-adjoint.

This is a good moment to give

Proof (Proof of Theorem 2) Everywhere in this proof $K_{\mathrm{op}}$ is considered as an operator with target space $\mathscr{H}_{K}$. We start from showing that condition (iii) of the theorem is equivalent to:

(bd) $\quad K_{\text {op }}: \ell_{\text {fin }}(X) \rightarrow \mathscr{H}_{K}$ is bounded.

Indeed, (iii) says that for some $c>0$ we have

$$
\sum_{x \in X} u(x) \overline{u(y)} K(x, y) \leq \delta \sum_{x \in X}|u(x)|^{2}
$$

for any $u \in \ell_{\text {fin }}(X)$, which is equivalent to (bd), as the left-hand side of (36) coincides with $\left\|K_{\mathrm{op}} u\right\|^{2}$. Now we turn to the main part of the proof.

Assume (iii) holds. Then the absolute value $B$ of the closure of $K_{\mathrm{op}}$ is positive and bounded (by (bd)). Observe that 


$$
\left\langle B e_{x}, B e_{y}\right\rangle_{\ell_{2}(X)}=\left\langle K_{\mathrm{op}} e_{x}, e_{y}\right\rangle_{\ell_{2}(X)}=K(x, y)
$$

and thus (e.g. by condition (c1) in Theorem 8) $K$ has a pdms root. Moreover, the above $B$ witnesses property (I) in Proposition 8 . So, it follows from item (II) of that proposition that all other possible pdms roots are in one-to-one correspondence with linear isometries $V: \overline{\mathcal{R}}(B) \rightarrow \ell_{2}(X)$ such that $V B$ is positive. But if $V$ is such an isometry, then $V B$ is self-adjoint (being positive and bounded) and it follows from the uniqueness of $B$ that $V B=B$ or, equivalently, that $V$ is the identity. This shows (i) - that is, that $K$ has a unique pdms root.

To prove the reverse implication, we assume that (iii) is false and we will show that (i) is false as well. To this end, assume $K$ has a pdms root (otherwise (i) does not hold). Let $B$ witness property (I) in Proposition 8 . We claim that $B$ is not bounded. Indeed, since (iii) does not hold, (bd) is false. And we infer from the proof of Lemma 4 that $K_{\mathrm{op}}=V B \mid \ell_{\text {lin }}(X)$ for some linear isometry $V: B\left(\ell_{\text {fin }}(X)\right) \rightarrow \mathscr{H}_{K}$. So, $B$ is not bounded as $K_{\mathrm{op}}$ is not such. Now it follows from Theorem 7 that there exists a positive (densely defined) operator $T$ in $\ell_{2}(X)$ such that $|T|=B \neq T$. Let $T=Q B$ be the polar decomposition of $T$. Then $Q \mid \overline{\mathcal{R}}(B)$ is isometric and differs from the identity map. So, there are at least two pdms roots of $K$ thanks to item (II) of Proposition 8.

Further, observe that it follows from the equivalence of (i) and (iii) (which we have already proved) that (ii) is implied by (i). So, it remains to show that if (iii) does not hold, then (ii) is false. To this end, assume there is no $c>0$ for which $K \ll c \delta_{X}$. Equivalently, $\mathscr{H}_{K} \not \subset \mathscr{H}_{\delta_{X}}=\ell_{2}(X)$ (cf. Lemma 5). So, there exists a unit vector $u \in \mathscr{H}_{K}$ such that $u \notin \ell_{2}(X)$. Then $L \stackrel{\text { def }}{=} \bar{u} \otimes u$ is a pd kernel such that $L \ll K$ (since $u$ is a unit vector in both $\mathscr{H}_{K}$ and $\mathscr{H}_{L}$-see, e.g., Corollary 2 on page 45 in [18]) and $\mathscr{H}_{L}=\operatorname{lin}\{u\}$ where

$$
\bar{u} \otimes u: X \times X \ni(x, y) \mapsto \overline{u(x)} u(y) \in \mathbb{C} .
$$

In particular, $\ell_{2}(X) \cap \mathscr{H}_{L}=\{0\}$ and hence $L$ does not have a pdms root (thanks to condition (d) of Theorem 8).

Remark 3 An inspection of the proofs of Theorems 7 and 2, combined with Proposition 1, shows that if a pd kernel has at least two pdms roots, then it actually has uncountably many such roots. We leave the details to interested readers.

Corollary 5 A pd kernel $K$ on $X$ has a unique pdms root iff $\mathscr{H}_{K} \subset \ell_{2}(X)$.

Proof The assertion immediately follows from Theorem 2 and Lemma 5.

Corollary 6 Let $K$ be a pd kernel on $X$ that has a unique pdms root. For any $p d$ kernel $L$ on $X, L$ has a pdms root iff so has $K+L$.

Proof The proof of Theorem 2 shows that $T \stackrel{\text { def }}{=} K_{\mathrm{op}}: \ell_{\text {fin }}(X) \rightarrow H \stackrel{\text { def }}{=} \mathscr{H}_{K}$ is bounded. Let $R: \ell_{\text {fin }}(X) \rightarrow E$ be any operator that factorizes $L$. Then $T \oplus R: \ell_{\text {fin }}(X) \rightarrow$ $H \oplus E$ factorizes $K+L$ (as $T$ factorizes $K$ ). Since $T$ is bounded, $R$ is closable iff so 
is $T \oplus R$. Thus, the conclusion follows from condition (c1) of Theorem 8 and Lemma 4.

Example 1 Simplest possible pd kernels on a set $X$ are of the form (37) where $u: X \rightarrow \mathbb{C}$ is totally arbitrary. (The simplicity of these kernels can be justified as follows: they are precisely those pd kernels $K$ for which $\operatorname{dim}\left(\mathscr{H}_{K}\right) \leq 1$.) Since $\mathscr{H}_{\bar{u} \otimes u}=\operatorname{lin}\{u\}$, we either have $\mathscr{H}_{\bar{u} \otimes u} \subset \ell_{2}(X)$ or $\ell_{2}(X) \cap \mathscr{H}_{\bar{u} \otimes u}=\{0\}$. So, condition (d) of Theorem 8 and Corollary 5 imply that for a function $v: X \rightarrow \mathbb{C}$ the following conditions are equivalent:

(i) $\bar{v} \otimes v$ has a pdms root;

(ii) $\bar{v} \otimes v$ has a unique pdms root;

(iii) $v \in \ell_{2}(X)$.

In Example 4 we will use the above characterization to give a (counter)example witnessing that the uniform limit of bounded pd kernels having a unique pdms root can have no pdms roots.

To simplify further statements, we introduce a few additional notions:

Definition 4 A pd kernel $K$ on $X$ is said to be

- diagonal if $K(x, y)=0$ for any distinct $x, y \in X$;

- pointwise countable if for any $x \in X$ the set $\{y \in X: K(x, y) \neq 0\}$ is (at most) countable.

A rescaling of $K$ is a pd kernel of the form

$$
X \times X \ni(x, y) \mapsto \overline{u(x)} u(y) K(x, y) \in \mathbb{C}
$$

where $u: X \rightarrow \mathbb{C}$ is arbitrary. The kernel given by (38) is called $u$-rescaling. The $u$ rescaling is non-vanishing if $u$ has no zeros.

Our nearest aim is to characterize those kernels that admit a non-vanishing rescaling having a pdms root. The following result will serve as a useful tool in investigating this issue. Everywhere below $\mathbb{N}$ denotes the set of all positive integers.

Lemma 7 Let $K$ be a pd kernel on $\mathbb{N}$ and $D$ be the diagonal pd kernel such that $D(n, n)=n^{2}$ for any $n$.

(I) If $\sum_{n, m=1}^{\infty}|K(n, m)|^{2} \leq 1$, then $K \ll \delta_{\mathbb{N}}$.

(II) If $\sum_{n=1}^{\infty} K(n, n) \leq 1$, then $K \ll \delta_{\mathbb{N}}$.

(III) If $|K|$ is upper bounded by $c>0$, then $K \ll \frac{c \pi^{2}}{6} D$.

Proof Although properties (I) and (II) are consequences of well-known results on Schatten class and Hilbert-Schmidt operators (consult, e.g., [19] or the material of $\S 18$ in [7]), below we present their brief proofs.

To show (I), for any $\alpha \in \ell_{\text {fin }}(\mathbb{N})$ the Schwarz inequality yields 


$$
\begin{aligned}
\sum_{n, m=1}^{\infty} \alpha(n) \overline{\alpha(m)} K(n, m) & \leq \sqrt{\sum_{n, m=1}^{\infty}|\alpha(n) \overline{\alpha(m)}|^{2}} \cdot \sqrt{\sum_{n, m=1}^{\infty}|K(n, m)|^{2}} \\
& \leq \sqrt{\sum_{n=1}^{\infty}|\alpha(n)|^{2} \cdot \sum_{m=1}^{\infty}|\alpha(m)|^{2}}=\sum_{n=1}^{\infty}|\alpha(n)|^{2}
\end{aligned}
$$

which is equivalent to $K \ll \delta_{\mathbb{N}}$. Further, (II) is a consequence of (I) since

$$
\sum_{n, m=1}^{\infty}|K(n, m)|^{2} \leq \sum_{n, m=1}^{\infty} K(n, n) K(m, m)=\left(\sum_{n=1}^{\infty} K(n, n)\right)^{2}
$$

(cf. the paragraph following Theorem 9). We turn to (III). For simplicity, denote the $u$-rescaling of a pd kernel $L$ by $L_{u}$. In particular, $\left(L_{u}\right)_{v}=L_{u v}$. Define $u: \mathbb{N} \ni n \mapsto \frac{\sqrt{6}}{\pi n \sqrt{c}} \in(0, \infty)$. Since $|K|$ is bounded by $c$ and $\sum_{n=1}^{\infty} n^{-2}=\frac{\pi^{2}}{6}$, we easily get that $\sum_{n=1}^{\infty} K_{u}(n, n) \leq 1$. So, we conclude from (II) that $K_{u} \ll \delta_{\mathbb{N}}$. Consequently, $K=\left(K_{u}\right)_{\frac{1}{u}} \ll\left(\delta_{\mathbb{N}}\right)_{\frac{1}{u}}=\frac{c \pi^{2}}{6} D$ and we are done.

Now we can characterize pd kernels admitting non-vanishing rescalings having pdms roots.

Theorem 10 For a pd kernel $K$ on $X$ the following conditions are equivalent:

(i) $K$ has a non-vanishing rescaling that has a pdms root;

(ii) the v-rescaling of $K$ has a unique pdms root for some $v: X \rightarrow(0,1)$;

(iii) $\quad K \ll D$ for some diagonal pd kernel $D$ on $X$;

(iv) $K \ll C$ for some pointwise countable pd kernel $C$ on $X$;

(v) $K$ is pointwise countable.

\section{In particular,}

- each pd kernel on a countable set satisfies conditions (ii) and (iii);

- the collection $\mathscr{C}$ of all pd kernels $K$ on $X$ that satisfy (i) is a convex cone such that $L \in \mathscr{C}$ whenever $0 \ll L \ll K$ for some $K \in \mathscr{C}$ or $L$ is the pointwise product of two members of $\mathscr{C}$.

Proof Since the additional claim of the theorem easily follows from the equivalence of (i), (iii) and (v) (recall that-according to the Schur's theorem [20] - the pointwise product, usually called the Hadamard product, of two pd kernels defined on a common set is a pd kernel as well; see also Sect. 3 of Chapter I in [9] - consult the material on page 9 therein), we only need to show the equivalence of all conditions (i)-(v). As done in the previous proof, we shall denote, for simplicity, the $u$-rescaling of a pd kernel $L$ by $L_{u}$. First we will show the following implications: (iii) $\Rightarrow($ ii) $\Rightarrow($ i $) \Rightarrow(v) \Rightarrow$ (iii) and then we will briefly deduce the equivalence of (iv) and (iii).

Assume (iii) holds and let $D$ be as specified therein. Let $v: X \rightarrow(0,1)$ be given by $v(x) \stackrel{\text { def }}{=} 1 / \max (\sqrt{D(x, x)}, 2)(x \in X)$. Since $K \ll D$, we get $K_{v} \ll D_{v}$ and easily 
$D_{v} \ll \delta_{X}$. Hence $K_{v} \ll \delta_{X}$ and it follows from Theorem 2 that $K_{v}$ (or $v$ ) witnesses (ii). Now observe that (i) implies (v) by condition (b) of Theorem 8 , and obviously follows from (ii). So, we now assume that (v) holds and will show that (iii) is fulfilled. First of all, observe that $K$, being pointwise countable, induces a decomposition of $X=\bigsqcup_{s \in S} X_{s}$ into at most countable (pairwise disjoint) sets $X_{s}$ such that $K=\bigoplus_{s \in S} K^{(s)}$ where $K^{(s)}=K \mid X_{s} \times X_{s}(s \in S)$ (cf. the proof that (b) follows from (a1) in Theorem 8). Since $X_{s}$ is countable, there exists a function $u_{s}: X_{s} \rightarrow(0,1)$ such that $\sum_{x \in X_{s}} u_{s}(x)^{2} K^{(s)}(x, x) \leq 1$. We infer from Lemma 7 that $\left(K^{(s)}\right)_{u_{s}} \ll \delta_{X_{s}}$. Consequently, $\bigoplus_{s \in S}\left(K^{(s)}\right)_{u_{s}} \ll \delta_{X}$. Now it suffices to define $u$ : $X \rightarrow(0,1)$ as the union of all $u_{s}$ (that is, $u\left\lceil X_{s}=u_{s}\right.$ ) and $D$ as $\left(\delta_{X}\right)_{\frac{1}{u}}$ to get $K=\left(K_{u}\right)_{\frac{1}{u}}=\left(\bigoplus_{s \in S}\left(K^{(s)}\right)_{u_{s}}\right)_{\frac{1}{u}} \ll\left(\delta_{X}\right)_{\frac{1}{u}}=D$.

It remains to explain why (iv) is equivalent to (iii). Since diagonal pd kernels are pointwise countable, (iv) follows from (iii). Conversely, if (iv) holds and $C$ is as specified therein, then we know that there exists a diagonal pd kernel $D$ such that $C \ll D$ (as $C$ satisfies (v)). Then clearly $K \ll D$ and we are done.

Property (VI) of Theorem 9 implies that if $K$ and $L$ are pd kernels on $X$ that have pdms roots, then the kernel $K+L$ has a pdms root as well. Conversely, a basic consequence of Theorem 2 is that if $K+L$ has a unique pdms root, so have both $K$ and $L$. It turns out that a counterpart of this property for pd kernels having at least two pdms roots is (in a sense always - see Proposition 9 below) false and when the kernel acts on a countable set, the property mentioned above crashes in a striking way (see item (A) in Proposition 9). To be more precise, we call a pd kernel free of pdms roots if no non-zero pd kernel $L \ll K$ has a pdms root. With the aid of the results of Sect. 3, we now show that

Proposition 9 Let $K$ be a pd kernel on $X$ that has at least two pdms roots.

(A) If $X$ is countable, $K$ is a sum of two pd kernels each of which is free of pdms roots.

(B) $K$ is a sum of two pd kernels none of which has a pdms root.

In the proof we shall make use of the following

Lemma 8 A pd kernel $K$ on $X$ is free of pdms roots iff $\ell_{2}(X) \cap \mathscr{H}_{K}=\{0\}$.

Proof Let $K_{0}$ be as specified in property (IX) listed in Theorem 9. Observe that $K$ is free of pdms roots iff $K_{0} \equiv 0$. An inspection of the proof of the aforementioned item (IX) shows that $\mathscr{H}_{K_{0}}$ coincides with the closure in $\mathscr{H}_{K}$ of $\ell_{2}(X) \cap \mathscr{H}_{K}$, from which the conclusion follows.

Proof (Proof of Proposition 9) We start from (A). It follows from the countability of $X$ that $\mathscr{H}_{K}$ is separable. Since $K$ has a pdms root, $\mathcal{R} \stackrel{\text { def }}{=} \ell_{2}(X) \cap \mathscr{H}_{K}$ is dense in $\mathscr{H}_{K}$ (by Theorem 8). Moreover, $\mathcal{R} \neq \mathscr{H}_{K}$, because $K$ has more than one pdms root (see Corollary 5). Note that $\mathcal{R}$ is an operator range in $\mathscr{H}_{K}$ (the proof of (X) in Theorem 9 shows that $\mathcal{R}$ admits a Hilbert space norm stronger than the norm 
induced from $\left.\mathscr{H}_{K}\right)$. So, condition (g) of Theorem 5 implies that there is a closed linear subspace $\mathscr{W}$ of $\mathscr{H}_{K}$ such that $\mathscr{W} \cap \mathcal{R}=\mathscr{W}^{\perp} \cap \mathcal{R}=\{0\}$. Equivalently,

$$
\mathscr{W} \cap \ell_{2}(X)=\mathscr{W}^{\perp} \cap \ell_{2}(X)=\{0\} .
$$

Denoting by $P$ and $Q$ the orthogonal projections in $\mathscr{H}_{K}$ onto, respectively, $\mathscr{W}$ and $\mathscr{W}^{\perp}$, we define pd kernels $K_{1}$ and $K_{2}$ on $X$ by

$$
K_{1}(x, y) \stackrel{\text { def }}{=}\langle P K(x, \cdot), P K(y, \cdot)\rangle_{\mathscr{H}_{K}}
$$

and $K_{2}(x, y) \stackrel{\text { def }}{=}\langle Q K(x, \cdot), Q K(y, \cdot)\rangle_{\mathscr{H}_{K}}$. Then $\mathscr{H}_{K_{1}}=\mathscr{W}$ and $\mathscr{H}_{K_{2}}=\mathscr{W}^{\perp}$ (see Theorem 5 on page 37 in [18]) and therefore (thanks to (39) and Lemma 8) both $K_{1}$ and $K_{2}$ are free of pdms roots. But $K_{1}+K_{2}=K$ (since $P+Q=I$ ) and we are done.

Now we pass to the general case (item (B)). We may and do assume $X$ is uncountable. We will show that $X$ contains a countable subset $A$ such that

$$
K=(K\lceil A \times A) \oplus(K \uparrow(X \backslash A) \times(X \backslash A))
$$

and $K\lceil A \times A$ has at least two pdms roots. Assume for a moment that we have already found such a set $A$. It then follows from (A) that

$$
K\left\lceil A \times A=L_{1}+L_{2}\right.
$$

where $L_{1}$ and $L_{2}$ are pd kernels on $A$ without pdms roots. We define pd kernels $K_{1}$ and $K_{2}$ on $X$ by:

$$
K_{1}=L_{1} \oplus(K \uparrow(X \backslash A) \times(X \backslash A))
$$

and $K_{2}=L_{2} \oplus 0$ (where 0 here means the zero function on $(X \backslash A) \times(X \backslash A)$ ). It is easily seen that both $K_{1}$ and $K_{2}$ are pd kernels such that $K=K_{1}+K_{2}$. Moreover, it follows from property (I) in Theorem 9 that none of $K_{1}$ and $K_{2}$ has a pdms root. (Indeed, if $K_{j}$ had a pdms root, so would have $K_{j}\left\lceil A \times A=L_{j}\right.$ which is impossible.) Hence, it remains to construct the set $A$ with appropriate properties. We will do this with the aid of condition (b) of Theorem 8 .

It follows from the aforementioned result that $K=\bigoplus_{s \in S} K_{s}$ where for any $s \in S$, $K_{s}$ is a pd kernel on a countable set $X_{s}$ that has a pdms root. If one of $K_{s}$ has at least two pdms roots, we just set $A=X_{s}$ and finish the proof. So, we assume that $K_{s}$ has a unique pdms root for any $s \in S$. It follows from Theorem 2 that

$$
K_{s} \ll c_{s} \delta_{X_{s}}
$$

for some constant $c_{s} \geq 0$. We take $c_{s}$ to be the smallest possible non-negative number witnessing (40). We claim that

$$
\sup _{s \in S} c_{s}=\infty
$$

Indeed, if $c \stackrel{\text { def }}{=} \sup _{s \in S} c_{s}$ was finite, then we would have $K_{s} \ll c \delta_{X_{s}}$ for all $s \in S$ and thus also $K \ll c \delta_{X}$, which would mean that $K$ would have a unique pdms root (by 
Theorem 2). So, (41) holds and therefore there exists a sequence $s_{1}, s_{2}, \ldots \in S$ for which $\lim _{n \rightarrow \infty} c_{s_{n}}=\infty$. We set $A \stackrel{\text { def }}{=} \bigcup_{n=1}^{\infty} X_{s_{n}}$. It follows from property (I) in Theorem 9 that $K\left\lceil A \times A\right.$ has a pdms root. Further, since the numbers $c_{s}$ have been chosen optimal, there is no $c>0$ such that $K\left\lceil A \times A \ll c \delta_{A}\right.$. Consequently, $K\lceil A \times A$ has more than one pdms root (by Theorem 2) and we are done.

Since any pd kernel defined on a finite set has a (unique) pdms root, condition (b) of Theorem 8 completely reduces the study of the class of pd kernels having pdms roots to pd kernels on infinite countable sets. So, pd kernels on $\mathbb{N}$ (or $\mathbb{Z}$ ) are of special interest. Below we give a single positive example on the existence of pdms roots and a list of counterexamples to related subjects. Everywhere below $\ell_{2}=$ $\ell_{2}(\mathbb{N})$ (that is, indices of sequences in $\ell_{2}$ start from 1 ), $\ell_{\text {fin }}=\ell_{\text {fin }}(\mathbb{N})$ and $e_{1}, e_{2}, \ldots$ is the canonical basis of $\ell_{2}$.

Example 2 Let $S \in \mathfrak{B}\left(\ell_{2}\right)$ be the standard unilateral shift (that is, $S$ is a linear isometry such that $\left.S e_{n}=e_{n+1}\right)$ and $f \in \ell_{2}$ be arbitrary. Then the pd kernel $K$ on $\mathbb{N}$ given by:

$$
K(n, m)=\left\langle S^{n-1} f, S^{m-1} f\right\rangle_{\ell_{2}} \quad(n, m \in \mathbb{N})
$$

has a pdms root. To prove this, it is sufficient to find a closable operator that factorizes $K$ (see item (c1) in Theorem 8). To this end, we model the shift $S$ on the Hardy space $H^{2}$ of holomorphic functions on the disc $\mathbb{D}=\{z \in \mathbb{C}:|z|<1\}$. Recall that:

$\left(H^{2}\right.$ a) a holomorphic function $u: \mathbb{D} \ni z \mapsto \sum_{k=0}^{\infty} a_{k} z^{k} \in \mathbb{C}$ belongs to $H^{2}$ if $\sum_{k=0}^{\infty}\left|a_{k}\right|^{2}<\infty$;

$\left(H^{2} \mathrm{~b}\right)$ the monomials $1, z, z^{2}, \ldots$ form an orthonormal basis of $H^{2}$ (we use here a standard simplified notation; $z^{k}$ is in fact the function $z \mapsto z^{k}$ restricted to $\mathbb{D}$ );

$\left(H^{2} \mathrm{c}\right) \quad$ for any $z \in \mathbb{D}$, the evaluation functional $H^{2} \ni u \mapsto u(z) \in \mathbb{C}$ is continuous (the reproducing kernel of $H^{2}$ has the form $\left.(z, w) \mapsto \frac{1}{1-\bar{z} w}\right)$;

$\left(H^{2} \mathrm{~d}\right) \quad$ the operator $M: H^{2} \rightarrow H^{2}$ given by $(M u)(z)=z u(z)\left(z \in \mathbb{D}, u \in H^{2}\right)$ is unitarily equivalent to $S$; more precisely, if $V: \ell_{2} \rightarrow H^{2}$ is a unitary operator such that $V e_{n}=z^{n-1}(n>0)$, then $V^{-1} M V=S$.

Now for $f \in \ell_{2}$ set $F \stackrel{\text { def }}{=} V f \in H^{2}$ and define $T: \operatorname{lin}\left\{z^{k}: k \geq 0\right\} \rightarrow H^{2}$ as the multiplication operator by $F$ (that is, $(T p)(z)=F(z) p(z)$ for $p \in \mathcal{D}(T)$ and $z \in \mathbb{D}$ ). It easily follows from the above property $\left(H^{2} \mathrm{c}\right)$ that $T$ is closable. Therefore $V^{-1} T V: \ell_{\text {fin }} \rightarrow \ell_{2}$ is closable as well. Note that $S^{k}=V^{-1} M^{k} V$ and hence $S^{k} f=V^{-1} M^{k} F=V^{-1} T z^{k}=V^{-1} T V e_{k+1}$. Consequently, $V^{-1} T V$ factorizes $K$ and we are done.

Remark 4 Continuing the notation introduced in Example 2, we can find a specific pdms root of the kernel $K$ (given by (42)) provided we are able to determine the polar decomposition of $\bar{T}$ (where $T$ is as specified in the previous example). Indeed, if $\bar{T}=Q B$ is the polar decomposition, then $L: \mathbb{N} \times \mathbb{N} \rightarrow \mathbb{C}$ given by $L(n, m)=$ 
$\left\langle B z^{n-1}, z^{m-1}\right\rangle_{H^{2}}$ is a pdms root of $K$-this claim easily follows from the proof of Theorem 8 that (c2) is implied by (c1) and (a1) by (c2).

Example 3 Similarly to the notion of an $\ell_{2}$-kernel, let us call a kernel $K$ on $\mathbb{N}$ a $c_{0^{-}}$kernel if $\lim _{n \rightarrow \infty}(|K(p, n)|+|K(n, p)|)=0$ for all $p \in \mathbb{N}$. Item (IV) in Theorem 9 says that each bounded pd kernel on $\mathbb{N}$ that has a pdms root is a $c_{0}$-kernel. On the other hand, pd $\ell_{2}$-kernels always have pdms roots. So, two natural (contrary) questions arise:

(Question A) Does every bounded pd $c_{0}$-kernel on $\mathbb{N}$ have a pdms root?

(Question B) Is every bounded pd kernel that has a pdms root an $\ell_{2}$-kernel?

In this example we answer these two questions in the negative. Both the kernels constructed below will be constant on the diagonal $\{(n, n): n \in \mathbb{N}\}$.

(A) In this part we construct a pd kernel $K$ on $\mathbb{N}$ that has a pdms root and satisfies:

- $\quad K(n, n)=1$ for all $n \in \mathbb{N}$ (and thus $K$ is bounded);

- $K(m, \cdot) \notin \bigcup_{p>0} \ell_{p}$ for any $m>0$.

Define a sequence $f=\left(a_{1}, a_{2}, \ldots\right)$ of positive real numbers as follows:

$$
a_{n}=\sqrt{\frac{1}{H_{n}}-\frac{1}{H_{n+1}}}=\frac{1}{\sqrt{(n+1) H_{n} H_{n+1}}}
$$

where $H_{n} \stackrel{\text { def }}{=} \sum_{k=1}^{n} \frac{1}{k}$. The second formula for $a_{n}$ shows that the sequence $f$ is monotone decreasing, whereas the first implies that the series $\sum_{k=1}^{\infty} a_{k}^{2}$ is telescoping and its sum equals $\frac{1}{H_{1}}=1$. So, $f \in \ell_{2}$. Let $S \in \mathfrak{B}\left(\ell_{2}\right)$ be the shift as specified in Example 2. That example shows that the pd kernel $K$ given by (42) has a pdms root. We now check that $K$ has all announced properties. It is easily seen that $K(n, n)=\|f\|^{2}=1$. Further, for any $n>0$ we have (recall that $f$ is monotone decreasing):

$$
K(1, n)=\sum_{k=1}^{\infty} a_{k} a_{k+n-1} \geq \sum_{k=1}^{\infty} a_{k+n-1}^{2}=\frac{1}{H_{n}} .
$$

Since $\lim _{n \rightarrow \infty} \frac{\log n}{H_{n}}=1$, we get that $\sum_{n=1}^{\infty} \frac{1}{H_{n}^{p}}=\infty$ for any $p>0$ and therefore $K(1, \cdot) \notin \bigcup_{p>0} \ell_{p}$. Finally, observe that $K(m, n)=K(1, n-m+1)$ whenever $n>m>0$ and thus $K(m, \cdot) \notin \bigcup_{p>0} \ell_{p}$ as well.

(B) In contrast to the example given in (A), now we construct a pd kernel $K$ : $\mathbb{N} \times \mathbb{N} \rightarrow[0,1]$ such that $K(m, \cdot) \in \bigcap_{p>2} \ell_{p}$ for any $m \in \mathbb{N}$, but $K$ has no pdms roots. To this end, we take any sequence $u \in\left(\bigcap_{p>2} \ell_{p}\right) \backslash \ell_{2}$ whose all entries lie in $[0,1]$ and define $K_{0}$ as $u \otimes u$ (see (37)). It follows from Example 1 that $K_{0}$ has no pdms roots. However, $K_{0}(m, \cdot)=u(m) u \in \bigcap_{p>2} \ell_{p}$. Now let $D$ be the diagonal pd kernel on $\mathbb{N}$ such that $D(n, n)=1-u(n)^{2}$. Since 
$D \ll \delta_{\mathbb{N}}$, we conclude that $D$ has a unique pdms root. So, it follows from Corollary 6 that $K \stackrel{\text { def }}{=} K_{0}+L$ has no pdms roots. This kernel satisfies $K(n, n)=$ 1 for all $n \in \mathbb{N}$ and $K(m, \cdot) \in \bigcap_{p>2} \ell_{p}$ for any $m>0$.

The above examples suggest that there is no handy description of bounded pd kernels that have a pdms root.

Example 4 Properties (VI) and (VII) listed in Theorem 9 suggest that perhaps pd kernels on a given set that have pdms roots form a set closed in the pointwise or uniform topology (in the space of all kernels). As the following simple example shows, this is not the case.

Let $u: \mathbb{N} \rightarrow[0,1]$ be given by $u(n)=\frac{1}{\sqrt{n}}$ and $K \stackrel{\text { def }}{=} u \otimes u$ (see (37)). We infer from Example 1 that $K$ has no pdms roots. Moreover, since $\operatorname{dim}\left(\mathscr{H}_{K}\right)=1, K$ is free of pdms roots. Now for $n>0$ let $K_{n}$ be a pd kernel on $\mathbb{N}$ such that $K_{n}(p, q)=K(p, q)$ if both $p$ and $q$ are less than $n$, and $K_{n}(p, q)=0$ otherwise. It is easy to see that $K_{n}$ is a pd kernel. Since $K_{n}$ is supported on a finite set, it has a unique pdms root (e.g., by Theorem 2). However, since $\lim _{n \rightarrow \infty} u(n)=0, K_{n}$ uniformly converge to $K$. So, the uniform limit of a bounded sequence of pd kernels each of which has a unique pdms root can be free of pdms roots.

We end the paper with the following

Example 5 Property (III) listed in Theorem 9 gives a necessary condition for an unbounded pd kernel to have a pdms root that reads as follows: if an unbounded pd kernel has a pdms root, so have all its bounded rescalings produced by bounded scalar functions. In this example we show that it is insufficient. More precisely, we will construct an unbounded pd kernel $K$ on $\mathbb{N}$ such that:

- each bounded rescaling of $K$ has a unique pdms root;

- $K$ has no pdms roots.

To construct such a kernel, it is sufficient to find two self-adjoint bounded operators $A, B \in \mathfrak{B}(H)$ on a separable Hilbert space $H$ such that for some orthonormal basis $f_{1}, f_{2}, \ldots$ of $H$ the following conditions hold:

(aux1) $\mathcal{R}(A) \cap \mathcal{R}(B)=\{0\} ;$

(aux2) $\quad A f_{n}=r_{n} f_{n}$ where $0<r_{n} \rightarrow 0$ as $n \rightarrow \infty$;

(aux3) $\quad\left\|B f_{n}\right\|=1$ for any $n>0$.

To convince oneself of that, assume we have found such operators $A$ and $B$. Observe that (aux2) implies that $\mathcal{N}(A)=\{0\}$ (and thus $\mathcal{R}(A)$ is dense in $H$ ). We set $\mathcal{D} \stackrel{\text { def }}{=} \mathcal{R}(A)$ and $C \stackrel{\text { def }}{=} A^{-1}: \mathcal{D} \rightarrow H$. Additionally, let $U: \ell_{2} \rightarrow H$ be the unitary operator such that $U e_{n}=f_{n}$. The kernel $K$ we search for is given by:

$$
K(n, m) \stackrel{\text { def }}{=}\left\langle B C U e_{n}, B C U e_{m}\right\rangle_{H}=\frac{\left\langle B f_{n}, B f_{m}\right\rangle_{H}}{r_{n} r_{m}} \quad(n, m \in \mathbb{N})
$$


(the latter formula follows from (aux2)). To show that $K$ has no pdms roots, it suffices to check that the operator $B C U\left\lceil\ell_{f i n}\right.$ is not closable (as $B C U$ factorizes $K$; see condition (c1) in Theorem 8 and Lemma 4). Since $U$ is unitary, we only need to verify that $T \stackrel{\text { def }}{=} B C\left\lceil\operatorname{lin}\left\{f_{n}: n>0\right\}\right.$ is not closable. To this end, first note that the graph $\Gamma(B C)$ of $B C$ is contained in the closure (in $H \times H$ ) of $\Gamma(T)$ (as $B$ is bounded and $\mathcal{D}(T)$ is a core for $C$ ). Thus, $T$ is closable iff so is $B C$, iff $(B C)^{*}$ is densely defined. But $(B C)^{*}=C^{*} B^{*}=C B$ (since $B$ is bounded and both $B$ and $C$ are selfadjoint) and $\mathcal{D}(C B)=\{0\}$ by (aux1). So, $T$ is not closable and hence $K$ has no pdms roots. Now let $v: \mathbb{N} \rightarrow \mathbb{C}$ be any function such that the $v$-rescaling of $K$ is bounded. This means that $\sup _{n \in \mathbb{N}}|v(n)|^{2} K(n, n)<\infty$. But $K(n, n)=\left(\frac{\left\|B f_{n}\right\|}{r_{n}}\right)^{2}=r_{n}^{-2}$ (by (aux3)). So, there exists $c>0$ such that

$$
\frac{|v(n)|}{r_{n}} \leq c \quad(n>0) .
$$

In particular, $\lim _{n \rightarrow \infty} v(n)=0$. Denote by $W$ the diagonal operator on $\ell_{2}$ (with respect to the orthonormal basis) such that $W e_{n}=\overline{v(n)} e_{n}$ ( $W$ is compact, but we do not need this property). Since

$$
\mathcal{D}=\left\{\sum_{n=1}^{\infty} \alpha_{n} f_{n}: \sum_{n=1}^{\infty}\left(\left|\alpha_{n}\right| / r_{n}\right)^{2}<\infty\right\},
$$

we easily infer from (43) that $\mathcal{R}(U W) \subset \mathcal{D}$ and $C U W$ is bounded (here we do not apply the Closed Graph Theorem-the boundedness of $C U W$ is a direct consequence of (43), (aux2) and the definitions of $C, U$ and $W$ ). Consequently, $Q \stackrel{\text { def }}{=} B A C W$ is bounded and

$$
\left\langle Q e_{n}, Q e_{m}\right\rangle_{H}=\overline{v(n)} v(m)\left\langle B C U e_{n}, B C U e_{m}\right\rangle_{H}=\overline{v(n)} v(m) K(n, m) .
$$

This means that the $v$-rescaling $L$ of $K$ is factorized by a bounded operator (namely, by $Q$ ), from which it easily follows that $L$ satisfies condition (iii) of Theorem 2 and therefore has a unique pdms root. In this way we have reduced our proof to the construction of self-adjoint operators $A$ and $B$ that satisfy (aux1)-(aux3), which we do below.

First of all, let $H$ stand for $L^{2}([0,1])$ (with the Lebesgue measure). We arrange all integers in a one-to-one sequence $k_{1}, k_{2}, \ldots$ and define $f_{1}, f_{2}, \ldots$ as a rearranged standard exponential orthonormal basis of $H: f_{n}(x)=e^{2 k_{n} \pi x i}$. Let $A \in \mathfrak{B}(H)$ be given by $A f_{n}=\frac{1}{n} f_{n}$. So, $A g=\sum_{n=1}^{\infty} \frac{\left\langle g, f_{n}\right\rangle_{H}}{n} f_{n}$ for any $g \in H$. Since the series $\sum_{n=1}^{\infty} \frac{\left\langle g \cdot f_{n}\right\rangle_{H}}{n}$ is absolutely convergent, we see that $\mathcal{R}(A)$ consists of continuous functions. Note also that (aux2) holds with $r_{n}=\frac{1}{n}$.

Now we will define $B$. To this end, take any bounded Borel function $u:[0,1] \rightarrow$ $(0, \infty)$ such that for any non-empty open interval $I \subset[0,1]$,

$$
\int_{I} \frac{d t}{u(t)^{2}}=\infty
$$


The proof that such a function exists is left to the reader. Additionally, we may and do assume that $u$ is a unit vector in $H$. We define $B$ as the multiplication operator by $u$; that is, $B: H \rightarrow H$ is given by $(B f)(t)=u(t) f(t)$. It is clear that $B$ is positive selfadjoint and bounded, and has trivial kernel. We claim that $\mathcal{R}(A) \cap \mathcal{R}(B)=\{0\}$. Indeed, it is sufficient to show that $\mathcal{R}(B)$ contains no non-zero continuous functions. To this end, assume that $g:[0,1] \rightarrow \mathbb{C}$ is continuous and non-zero. Then we can find a non-empty open interval $I \subset[0,1]$ and a constant $\varepsilon>0$ such that $|g(x)| \geq \varepsilon$ for all $x \in I$. But then $\int_{0}^{1}\left(\frac{|g(x)|}{u(x)}\right)^{2} d x \geq \int_{I} \frac{\varepsilon^{2}}{u(x)^{2}} d x=\infty$ (by (44)) and hence $\frac{g}{u} \notin H$. We conclude that $g \notin \mathcal{R}(B)$. Finally, since $\|u\|=1$ and $\left|f_{n}(x)\right|=1$ for any $x \in I$, condition (aux3) also holds and the proof is complete.

Open Access This article is licensed under a Creative Commons Attribution 4.0 International License, which permits use, sharing, adaptation, distribution and reproduction in any medium or format, as long as you give appropriate credit to the original author(s) and the source, provide a link to the Creative Commons licence, and indicate if changes were made. The images or other third party material in this article are included in the article's Creative Commons licence, unless indicated otherwise in a credit line to the material. If material is not included in the article's Creative Commons licence and your intended use is not permitted by statutory regulation or exceeds the permitted use, you will need to obtain permission directly from the copyright holder. To view a copy of this licence, visit http:// creativecommons.org/licenses/by/4.0/.

\section{References}

1. Alonso, A., Simon, B.: The Birman-Kreǐn-Vishik theory of self-adjoint extensions of semibounded operators. J. Oper. Theory 4, 251-270 (1980)

2. Aronszajn, N.: Theory of reproducing kernels. Trans. Am. Math. Soc. 68, 337-404 (1950)

3. Bergmann, S.: Über die Entwicklung der harmonischen Funktionen der Ebene und des Raumes nach Orthogonalfunktionen. Math. Ann. 86, 238-271 (1922)

4. Bergman, S.: The Kernel Function and Conformal Mapping. American Mathematical Society, Providence (1950)

5. Berlinet, A., Thomas-Agnan, Ch.: Reproducing Kernel Hilbert Spaces in Probability and Statistics. Kluwer, Dordrecht (2004)

6. Conway, J.B.: A Course in Functional Analysis. Springer, New York (1990)

7. Conway, J.B.: A Course in Operator Theory. American Mathematical Society, Providence (2000)

8. Dixmier, J.: Étude sur les variétés et les opérateurs de Julia. Bull. Soc. Math. Fr. 77, 11-101 (1949)

9. Donoghue Jr., W.F.: Monotone Matrix Functions and Analytic Continuation. Springer, Berlin (1974)

10. Douglas, R.G.: On majorization, factorization and range inclusion of operators in Hilbert space. Proc. Am. Math. Soc. 17, 413-416 (1966)

11. Fillmore, P.A., Williams, J.P.: On operator ranges. Adv. Math. 7, 254-281 (1971)

12. Friedrichs, K.: Spektraltheorie halbeschränkter Operatoren und Anwendung auf die Spektralzerlegung von Differential-Operatoren. Math. Ann. 109, 465-487 (1934)

13. Gerard, P., Pushnitski, A.: An inverse problem for self-adjoint positive Hankel operators. Int. Math. Res. Not. IMRN 13, 4505-4535 (2015)

14. Kato, T.: On finite-dimensional perturbations of self-adjoint operators. J. Math. Soc. Jpn. 9, 239-249 (1957)

15. Megretskii, A.V., Peller, V.V., Treil, S.R.: The inverse spectral problem for self-adjoint Hankel operators. Acta Math. 174, 241-309 (1995)

16. Paulsen, V.I., Raghupathi, M.: An Introduction to the Theory of Reproducing Kernel Hilbert Spaces. Cambridge University Press, Cambridge (2016)

17. Rosenblum, M., Rovnyak, J.: Hardy Classes and Operator Theory. Oxford University Press, New York (1985)

18. Saitoh, S.: Theory of Reproducing Kernels and Its Applications. Longman Scientiuc and Technical, Essex (1988) 
19. Schatten, R.: Norm Ideals of Completely Continuous Operators. Springer, Berlin (1960)

20. Schur, J.: Bemerkungen zur Theorie der beschränkten Bilinearformen mit unendlich vielen Veränderlichen. J. Reine Angew. Math. 140, 1-28 (1911)

21. Szafraniec, F.H.: Przestrzenie Hilberta z jądrem reprodukującym (Polish). Wydawnictwo Uniwersytetu Jagiellońskiego, Kraków (2004)

22. Szafraniec, F.H.: The reproducing kernel property and its space: the basics. In: Alpay, D. (ed.) Operator Theory, vol. I, pp. 3-30. Springer, Basel (2015)

23. Szafraniec, F.H.: The beginnings of the reproducing property: Bergman, Szegö, Bochner-or perhaps Zaremba after all (Polish). Wiad. Mat. 52, 53-67 (2016)

24. Weidmann, J.: Linear Operators in Hilbert Spaces. Springer, New York (1980)

25. Zaremba, S.: L'équation biharmonique et une classe remarquable de fonctions fondamentales harmoniques. Bulletin International de l'Académie des Sciences de Cracovie, pp. 147-196 (1907) 